\title{
Violation of generalized fluctuation-dissipation theorem in biological limit cycle oscillators with state-dependent internal drives: Applications to hair cell oscillations
}

\author{
Janaki Sheth $\odot,{ }^{1}$ Dolores Bozovic, ${ }^{1,2}$ and Alex J. Levine ${ }^{1,3,4}$ \\ ${ }^{1}$ Department of Physics and Astronomy, UCLA, Los Angeles, California 90095-1596, USA \\ ${ }^{2}$ California NanoSystems Institute, UCLA, Los Angeles, California 90095-1596, USA \\ ${ }^{3}$ Department of Chemistry and Biochemistry, UCLA, Los Angeles, California 90095-1596, USA \\ ${ }^{4}$ Department of Computational Medicine, UCLA, Los Angeles, California 90095-1596, USA
}

(Received 24 May 2020; accepted 20 April 2021; published 27 May 2021)

\begin{abstract}
The spontaneously oscillating hair bundle of sensory cells in the inner ear is an example of a stochastic, nonlinear oscillator driven by internal active processes. Moreover, this internal activity is state dependent in nature-it measures the current state of the system and changes its power input accordingly. We study the breakdown of two fluctuation-dissipation relations in these nonequilibrium limit cycle oscillators with and without state-dependent drives. First, in the simple model of the hair cell oscillator where the internal drive of the system is state independent, we observe the expected violation of the well-known, equilibrium fluctuation-dissipation theorem (FDT), and verify the existence of a generalized fluctuation-dissipation theorem (GFDT). This generalized theorem is analogous to one proposed earlier by Seifert and Speck. It requires the system to be analyzed in the co-moving frame associated with the mean limit cycle of the stochastic oscillator. We then demonstrate, via numerical simulations and analytic calculations, that in the presence of a state-dependent drive, the dynamical hair cell model violates both the FDT and this GFDT. We go on to show, using stochastic, finite-state, dynamical models, that such a drive in stochastic limit cycle oscillators generically violates both the FDT and GFDT. We propose that one may in fact use the breakdown of this particular GFDT as a tool to more broadly look for and quantify the effect of state-dependent drives within (nonequilibrium) biological dynamics.
\end{abstract}

DOI: 10.1103/PhysRevResearch.3.023150

\section{INTRODUCTION}

Biology is replete with nonequilibrium systems that expend energy to maintain cyclic steady-state dynamics. Examples include the chemical networks underlying circadian rhythms, activity patterns in neuronal networks, and cardiac rhythmogenesis [1-6]. The inner ear provides another striking example of such dynamics, for it contains an internal active amplifier that allows the auditory system to detect nanoscale displacements [7-9]. In a quiet environment, the inner ear can moreover generate spontaneous otoacoustic emissions, which are metabolically sensitive, indicating the presence of an internal oscillatory instability necessitating an energy source. Beyond its innate importance to the understanding of sensory neuroscience, the auditory system provides an experimentally tractable substrate in which to study nonequilibrium fluctuation-dissipation theorems. In this work, we explore one of the several previously proposed generalizations of the standard fluctuation-dissipation theorem, and test it in theoretical models of inner ear dynamics.

An integral part of the vertebrate peripheral auditory system, hair cells of the inner ear [10] transduce mechanical

Published by the American Physical Society under the terms of the Creative Commons Attribution 4.0 International license. Further distribution of this work must maintain attribution to the author(s) and the published article's title, journal citation, and DOI. displacements imposed by the incoming pressure waves into electrical signals. This process is mediated by direct mechanical gating, as specialized ion channels open in response to minute lateral deflections of the stereovilli [11]—columnar structures protruding from the hair cells' apical surface and interconnected by tip links. When a hair bundle is deflected by an incoming sound, motion as small as a few ångströms leads to an increase in the tension exerted on the tip links and hence the opening of the transduction channels. Furthermore, hair cells demonstrate a number of adaptation processes that are key in maintaining this exquisite sensitivity $[12,13]$. While the biophysical mechanisms behind their internal activity are not entirely known, a number of myosin motor species have been implicated in hair cells of different species, including Myosin 1C. These molecular motors climb along the internal actin filaments, and are believed to be connected to the tip links, thus providing a mechanism capable of continually adapting to incoming sounds and maintaining the optimal tension required for sensitive detection [14].

The mechanical feedback loop between the myosin motors and displacements of the stereovilli has important consequences for hair bundle dynamics. It allows for an unstable dynamical regime in which the bundle responds to mechanical input like a spring with a negative spring constant $[15,16]$. In this regime, the bundle undergoes active oscillations even in the absence of incoming pressure waves due to the active feedback between motor activity and bundle displacement. Since that endogenous drive depends on the deflections of the bundle, i.e., on the state of our biological system, it provides a 
direct example of adaptive control of a nonequilibrium steady state. We note that similar examples of adaptation are found in a number of biological systems, including cellular regulation [17] and bacterial chemosensing [18].

Biological systems are generally noisy, due to the thermal fluctuations of their constituent elements. Hair cells encounter stochasticity from a number of sources. Since they are immersed in a fluid environment, Brownian motion leads to fluctuations of the stereovilli on the order of a nanometer [19]. Force fluctuations resulting from stochastic myosin motor activity are also present and may contribute colored noise [19]. Finally, the membrane potential of the cell body, which influences hair bundle motility [20], fluctuates due to ion channel clatter and shot noise in the ionic transport through transmembrane channels [21]. As a result, the limit cycle oscillations of the hair cell bundle are innately noisy and thus provide a window into the basic nonequilibrium statistical mechanics of a noisy limit cycle oscillator. Further, they operate under adaptive control where the internal drive maintaining the nonequilibrium steady state responds to the oscillator's state.

Spontaneous oscillations of the hair bundle have been studied experimentally by direct measurements performed in vitro on preparations of the amphibian sacculus [7,15,22]. Based on these experiments, a set of robust mathematical models of the active oscillations has been developed, comprised of nonlinear differential equations of varying degrees of complexity [19,22,23]. The simplest model that captures the essential phenomena is a two-dimensional dynamical system that undergoes a supercritical Hopf bifurcation to the limit cycle (oscillatory) state [24-26]. In this manuscript, we use the stochastic normal form equation for the Hopf bifurcation to model the spontaneously oscillating state of the hair bundle, in order to study fluctuation-dissipation theorems associated with noisy nonequilibrium systems.

It is well known that the equilibrium fluctuation-dissipation theorem can fail in nonequilibrium steady states. In fact, the breakdown of the standard fluctuation-dissipation theorem (FDT) [27] has been used as a way to characterize the nonequilibrium nature of cytoskeletal networks [28]. Recently, a growing body of work has proposed multiple generalizations of this theorem, applicable to different classes of nonequilibrium steady states [29-33]. Baiesi and co-workers provide a general and formal derivation [34,35], applications of which include driven colloidal systems and living systems containing molecular motors [36]. In addition to the equilibrium FDT [27], we focus on the generalized FDT (GFDT) of Speck and Seifert [29,31]. Specifically, we use the GFDT to study a particular subclass of nonequilibrium steady-state systems-driven, limit cycle oscillators. We show that, as expected, the two-dimensional driven hair bundle model violates the standard, equilibrium FDT [37], but does obey the Seifert-Speck GFDT.

We find that this agreement with the GFDT, however, is predicated on the internal drive being state-independent. We define a state-dependent drive as one which measures the system's current state and responds predictably by modifying the system's internal power input. In the case of hair cell oscillators, this modulation of the power input may be instantiated by the slow dynamics of the myosin motors, calcium-mediated modulation of internal springs, or a combination of processes within the stereocillium. The concept, however, is much more general.

A state-dependent drive in a biological system is necessary but not sufficient for the homeostatic maintenance of a nonequilibrium state. Adaptation, as discussed in Refs. [14,18], requires that the drive return the system to its initial state following an exogenous perturbation. As we examine below, the nonisochronous Hopf model of hair cell dynamics features a state-dependent drive, but this drive is not adaptive in that the changes in the power generated by the drive do not return the oscillator to its unperturbed state after cessation of the external force.

Once we incorporate the state-dependent drive into our dynamical model, we observe violations of the nonequilibrium GFDT. Thus, we propose that just as the violation of the original FDT in biological systems is an important quantitative measure of nonequilibrium dynamics [38], violation of the nonequilibrium GFDT provides a quantitative indicator of the presence of an internal state-dependent drive in biological dynamical systems.

\section{THE STOCHASTIC HOPF OSCILLATOR}

The simplest dynamical model of hair bundle oscillations is the stochastic, supercritical Hopf oscillator in its normal form. This two-dimensional dynamical system can be described in terms of a complex variable $z(t)=x(t)+i y(t)$, which obeys the differential equation

$$
\dot{z}=z(\mu+i \omega)-b z|z|^{2}+\eta_{z}(t)+f_{z}(t),
$$

where $f_{z}(t)$ is an external deterministic force acting on this overdamped system, and $\eta_{z}(t)$ is a stochastic force, described below. The dynamics of the deterministic and unforced system $\left(f_{z}=\eta_{z}=0\right)$ are governed by the values of the model parameters $\left\{\mu, \omega, b=b^{\prime}-i b^{\prime \prime},\left(b^{\prime}, b^{\prime \prime}>0\right)\right\}$. The real parameter $\mu$ is the control parameter of the system. When $\mu<0$, this term gives rise to overdamped oscillations, leaving the system with an infinite basin of attraction about the single fixed point at $z=0$. At $\mu=0$, the oscillator undergoes a supercritical Hopf bifurcation. As this parameter becomes positive, the oscillator's dynamics describe a circular limit cycle of radius $R_{0}=$ $\sqrt{\mu / b^{\prime}}$, which also has an infinite basin of attraction. The limit cycle has an angular frequency given by $\omega_{0}=\omega+R_{0}^{2} b^{\prime \prime}$, where we assume that $\omega$ is real.

To specify the stochastic system, we include a Gaussian white noise force $\eta_{z}=\eta_{x}+i \eta_{y}$ with a zero mean:

$$
\begin{gathered}
\left\langle\eta_{i}(t)\right\rangle=0, \\
\left\langle\eta_{i}(t) \eta_{j}\left(t^{\prime}\right)\right\rangle=A_{i j} \delta\left(t-t^{\prime}\right),
\end{gathered}
$$

with the symmetric and diagonal matrix $A_{i j}\left(A_{x y}=0\right)$ allowing for the uncorrelated noise in the $x$ and $y$ channels to be drawn, in principle, from different Gaussian distributions. Finally, we include deterministic external perturbations via $f_{z}(t)=f_{x}(t)+i f_{y}(t)$.

In the following, it will be convenient to work in polar coordinates: $r=\sqrt{x^{2}+y^{2}}$ and $\phi=\arctan (y / x)$ [39]. Trajectories derived from Eq. (1) are those of an overdamped particle moving in two dimensions in response to a force field $\mathbf{f}$, which 


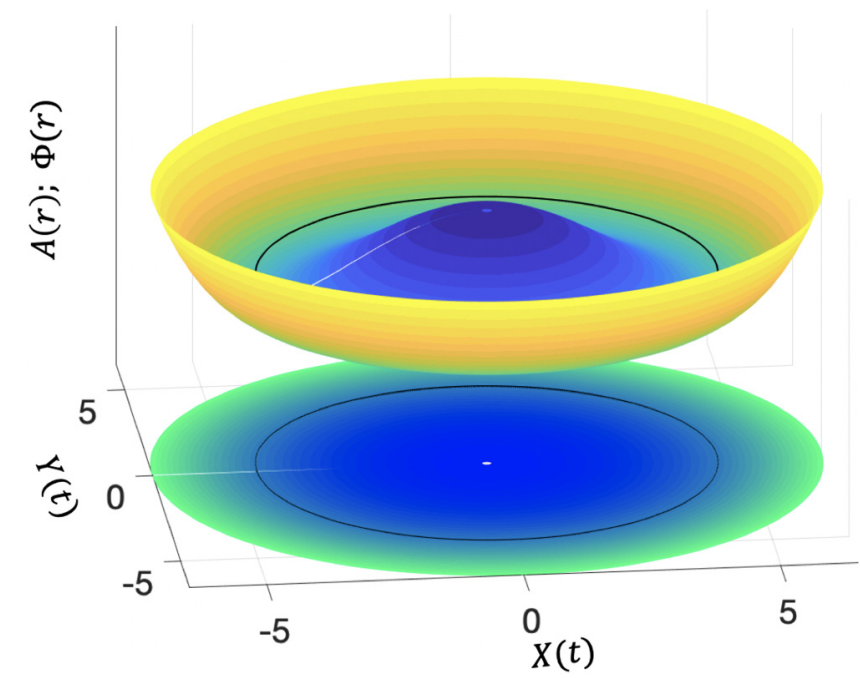

FIG. 1. The deterministic, unforced Hopf limit cycle (black curve) of radius $R_{0}$ sits in the azimuthally symmetric minimum potential region of $\Phi(\mathbf{r})$ as defined in Eq. (4) and is driven by the curl of $\mathbf{A}(\mathbf{r})$ given in Eq. (5). The color map for the three-dimensional $\Phi(\mathbf{r})$ runs from dark blue $(r=0)$ to yellow $(r=7)$. The magnitude of the vector potential $A(\mathbf{r})$ is shown as a colored disk which varies from dark blue $(r=0)$ to light green $(r=7)$.

can be decomposed into the gradient of an azimuthally symmetric scalar potential $\Phi(\mathbf{r})$ and the curl of a vector potential $\mathbf{A}=\hat{z} A(\mathbf{r})$, where

$$
\begin{aligned}
& \Phi(\mathbf{r})=-\frac{\mu}{2} r^{2}+\frac{b^{\prime}}{4} r^{4}, \\
& A(\mathbf{r})=-\frac{\omega}{2} r^{2}-\frac{b^{\prime \prime}}{4} r^{4} .
\end{aligned}
$$

Here, $\Phi(\mathbf{r})$ is the well-known "wine bottle" potential and is illustrated in Fig. 1 along with $\mathbf{A}(\mathbf{r})$. Also shown is the particle's deterministic, limit cycle. The curl of its vector potential, $\mathbf{f}_{\mathbf{v}}=\boldsymbol{\nabla} \times \mathbf{A}(\mathbf{r})$, is a constant azimuthal nonconservative force that drives the particle circularly along the minimum of $\Phi(\mathbf{r})$. Generating the only nonpotential force in the system, it is solely responsible for the internal power necessary to maintain the stable limit cycle and will henceforth be referred to as the internal drive of the Hopf oscillator. For the plots in Fig. 1, we use $\mu=40, \omega=10, b^{\prime}=2$, and $b^{\prime \prime}=2$.

When driven by white noise, the conservative system with $\omega=b^{\prime \prime}=0$ corresponds to the case of an overdamped particle in thermal equilibrium at some finite temperature. The vector potential, representing the action of the hair cell's endogenous molecular motors, does work on the overdamped system, generating the limit cycle oscillations, as shown in Fig. 2. We use the same parameter values as above. Other simulation details are described in Appendix A.

The appearance of a force field produced by a vector potential does not alone generate a limit cycle or even a nonequilibrium steady state. The necessary and sufficient conditions to create such a state with a time-independent force field are that (1) the force field is proportional to the curl of a vector potential, and (2) the nonconservative force does work on the particle that represents the state of the oscillator. A classic counterexample, where the second condition is not

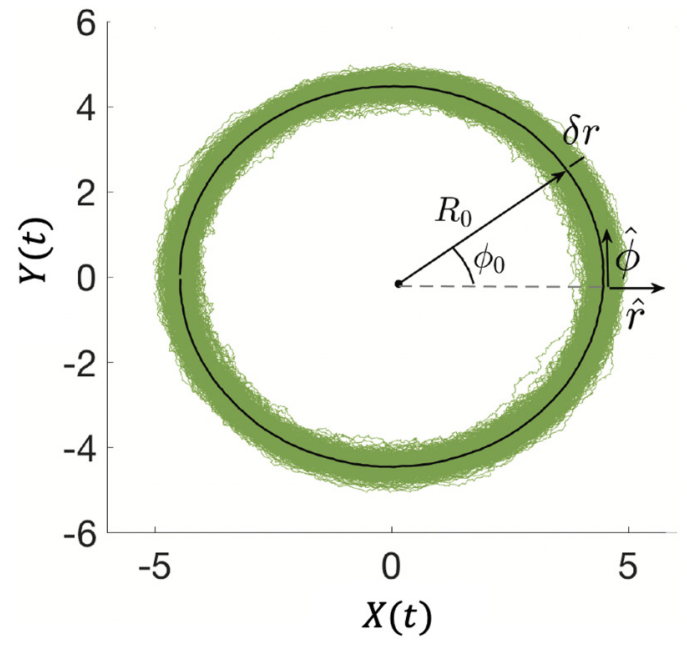

FIG. 2. A typical stochastic trajectory of a noisy Hopf oscillator based on Eq. (1) is shown in green. Its mean limit cycle is shown as the black circle, which has a particularly simple Frenet frame $\{\hat{r}, \hat{\phi}\}$ - these unit vectors denote the local normal and tangent to the curve, respectively.

met, is provided by a charged particle in a magnetic field. In Appendix B, we review this case, showing that a damped, charged particle in a two-dimensional harmonic potential and in a uniformly applied magnetic field, aligned in the direction perpendicular to the plane of the charged particle's motion, obeys the standard FDT.

Generally, for hair cell models, one allows the $b$ coefficient to be complex, as mentioned above. In this case, the azimuthal dynamics are of the form $\dot{\phi}=r^{-1}(\nabla \times \mathbf{A})=\omega+b^{\prime \prime} r^{2}$. The internal drive now depends on the state of the system, here given by $r$. In the dynamical systems literature, when the azimuthal coordinate is driven independently of the state i.e., when $b^{\prime \prime}=0$, the system is said to experience isochronous driving. Conversely, state-dependent driving where $b^{\prime \prime} \neq 0$ is referred to as nonisochronous. For our purposes, the important feature of this model is that the state-dependent nature of the drive underlying the steady-state limit cycle oscillations can be continuously varied through the one model parameter $b^{\prime \prime}$.

To study the fluctuations of the system about its limit cycle (when $\mu>0$ ), we expand about the limit cycle,

$$
\begin{gathered}
r(t)=R_{0}+\delta r(t), \\
\dot{\phi}(t)=\omega_{0}+\delta \dot{\phi}(t),
\end{gathered}
$$

to find two coupled stochastic linear Langevin equations for the fluctuations of the radius $\delta r$ and phase $\delta \phi$ of the oscillator,

$$
\begin{gathered}
\delta \dot{r}=-2 \mu \delta r+\eta_{r}+f_{r}, \\
\delta \dot{\phi}=2 b^{\prime \prime} \sqrt{\frac{\mu}{b^{\prime}}} \delta r+\eta_{\phi}+f_{\phi} .
\end{gathered}
$$

Here, the terms $\left\{\eta_{r}, \eta_{\phi}\right\}$ and $\left\{f_{r}, f_{\phi}\right\}$ are projections of the stochastic and perturbative forces onto the local normal $\hat{r}$ and tangent $\hat{\phi}$, respectively. These unit vectors span the FrenetSerret frame associated with the averaged limit cycle of the oscillator, being the local normal and tangent directions, 
respectively (see Fig. 2). The details of this averaging are given in Appendix A. The term $\sim b^{\prime \prime}$ in Eq. (9) is particularly important.

For nonvanishing $b^{\prime \prime}$, the power input by the drive into the linearized system of stochastic equations (linearized about the Frenet frame of the averaged limit cycle) varies linearly with deviations of the radius $r$ from its mean limit cycle value $R_{0}$. Writing $\delta r=r-R_{0}$ and the azimuthal force measured on the averaged limit cycle as $f_{v}$, we find the variations in power input to be given by

$$
\delta P=f_{v} \cdot \delta \dot{\phi}=2 b^{\prime \prime}\left(R_{0} \omega_{0}+b^{\prime \prime} R_{0}^{3}\right) R_{0} \delta r .
$$

Thus, when the stochastic system is near its limit cycle, the power input from the state-dependent drive increases when the radius exceeds its mean limit cycle value and decreases when that radius is less than that value. This state-dependent feature of the drive is essential for invalidating the GFDT, and thereby providing a window into potential feedback-dependent drives in this particular class of nonequilibrium steady-state systems (NESS).

The Frenet-Serret frame advances and simultaneously rotates along the stochastic oscillator's mean limit cycle at constant angular velocity $\omega_{0}$-which is also the value of the deterministically driven Hopf particle's local mean velocity. By working in this co-moving reference frame, we effectively subtract away the mean nonequilibrium dynamics of the steady-state oscillator (derivation in Appendix C). Doing so allows us to recover the GFDT for the nonequilibrium system, as discussed by Seifert and co-workers [29,31]. The use of the dimensionless phase angle $\phi$ instead of the arclength variable $s=R_{0} \phi$ requires the noise amplitudes $\eta_{r, \phi}$ to have different length dimensions. To account for this explicitly, we set second moments of the Gaussian force fluctuations in the frequency domain (given by $v$ ) to be

$$
\begin{gathered}
\left\langle\left|\eta_{r}(v)\right|^{2}\right\rangle=1, \\
\left\langle\left|\eta_{\phi}(v)\right|^{2}\right\rangle=R_{0}^{-2},
\end{gathered}
$$

which also has the effect of setting the effective noise temperature to $1 / 2$, since the mobilities in the Hopf equation have been set to unity. To account for this dimensional difference, it will be convenient in the following to define a symmetric "temperature matrix" by $\mathcal{T}_{r r}=1, \mathcal{T}_{r \phi}=R_{0}^{-1}, \mathcal{T}_{\phi \phi}=R_{0}^{-2}$. This choice of coordinates has no other consequences for our analysis.

To verify the GFDT in the co-moving frame, we first compute the correlation matrix of the linearized variables in the frequency domain,

$$
\mathcal{C}(v)=\left[\begin{array}{cc}
\left\langle|\delta r(v)|^{2}\right\rangle & \langle\delta r(v) \delta \phi(-v)\rangle \\
\langle\delta \phi(v) \delta r(-v)\rangle & \left\langle|\delta \phi(v)|^{2}\right\rangle
\end{array}\right] .
$$

Using Eqs. (8) and (9), we obtain

$$
\mathcal{C}(v)=\left[\begin{array}{cc}
\frac{1}{4 \mu^{2}+v^{2}} & 0 \\
0 & \frac{b^{\prime}}{\mu v^{2}}
\end{array}\right]+\frac{2 b^{\prime \prime}}{v\left(4 \mu^{2}+v^{2}\right)}\left[\begin{array}{cc}
0 & -i \\
i & \frac{2 b^{\prime \prime} \mu}{b^{\prime} v}
\end{array}\right] .
$$

The radial autocorrelations are those of an overdamped harmonic oscillator, as expected from the form of the scalar potential in Eq. (4), calculated near the circular limit cycle $r=R_{0}$. Similarly, the autocorrelations of the phase angle vary,
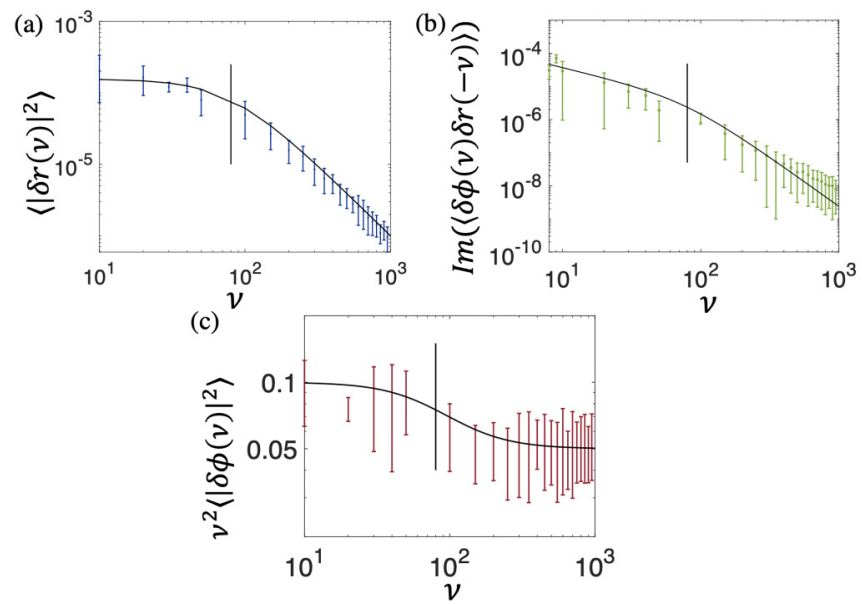

FIG. 3. Measured two-point correlations (colored points) of fluctuations $\delta r(v), \delta \phi(v)$ of the simulated stochastic Hopf limit cycle oscillator are shown in the frequency domain along with their corresponding analytic calculations (black lines)—see Eq. (14). Error bars show the standard deviation of the mean. (c) The frequency-dependent phase diffusion constant. The state-dependent drive introduces $\delta r, \delta \phi$ cross correlations (b) so that the radial fluctuations enhance phase diffusion for frequencies below the Lorentzian corner frequency of the radial fluctuations, indicated by the vertical (black) line in all the panels.

$\sim v^{-2}$, as expected for phase diffusion. When the system's internal drive is independent of its state or is isochronous $\left(b^{\prime \prime}=\right.$ $0)$, there is a simple, frequency-independent phase diffusion constant, and there are no cross correlations between the radial and phase fluctuations. A state-dependent drive, however, introduces both a frequency-dependent phase diffusion constant (observed in hair cell data [24]) and, more importantly, new cross correlations between the radial and phase fluctuations. Both of these effects arise because the power generated by the internal drive changes in response to the state of the system, given by $\delta r$, as discussed above.

All three correlation functions are shown in Fig. 3, where the solid (black) lines show the theoretical predictions, and the (colored) points show the numerical results from our Brownian simulations. The error bars on the numerical data points represent the standard deviation of the mean.

In order to test the validity of the GFDT, we need to compare the correlation functions computed from Eq. (14) with the linear response functions of the system. A direct calculation of these linear response functions,

$$
x_{\alpha}(v)=\chi_{\alpha \gamma}(v) f_{\gamma}(v),
$$

generates the matrix

$$
\chi(\nu)=\left[\begin{array}{cc}
\frac{1}{2 \mu-i v} & 0 \\
-2 b^{\prime \prime} \sqrt{\frac{\mu}{b^{\prime}}} \frac{1}{(i v)(2 \mu-i v)} & -\frac{1}{i \nu}
\end{array}\right] .
$$

Now, we may compare the correlation functions from Eq. (14) to the response functions from Eq. (16) to get the validity of the GFDT. Since we are working in the co-moving reference frame that zeros out the mean probability current, we expect the fluctuation-dissipation theorem of Seifert and Speck [29,31] to hold, at least in the limit of the state-independent 

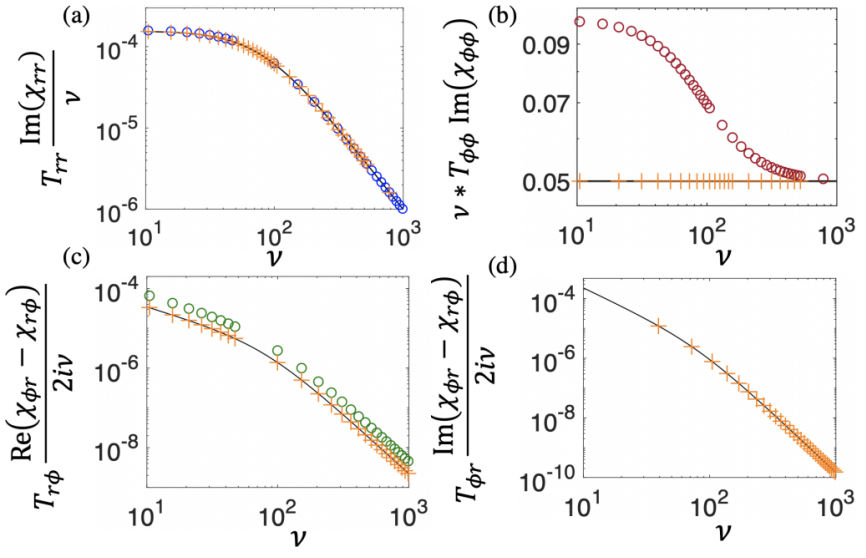

FIG. 4. Breakdown of GFDT in the Hopf oscillator with a statedependent drive. We compare the measured two-point correlations of Fig. 3 (circles) with those inferred from the numerical response function data via GFDT (orange crosses). The latter agrees with the analytical calculations (black lines) of $\left[\chi_{\alpha \beta}(v)-\chi_{\beta \alpha}(-v)\right] \mathcal{T}_{\beta \gamma}$ using Eq. (16). (a) While the GFDT predicted correlation function agrees with observations for the radial fluctuations, it (b) differs from those for the phase diffusion. In the bottom panels, we show the (c) real and (d) imaginary parts of $\chi_{\phi r}(v)-\chi_{r \phi}(-v)$. The former is related to the cross correlations of $\delta r$ and $\delta \phi$. The GFDT prediction of these correlations also fails (orange crosses vs green circles).

drive. Specifically, we expect that

$$
\left[\chi_{\alpha \beta}(v)-\chi_{\beta \alpha}(-v)\right]=\frac{2 i v}{\mathcal{T}_{\beta \gamma}} \mathcal{C}_{\alpha \gamma}(v)
$$

holds. To quantify any deviations from this equality produced by the state-dependent nature of the drive, it is convenient to define a deviation matrix $\Delta_{\alpha \beta}$ by subtracting the left side of Eq. (17) from the right (and multiplying through by the temperature matrix):

$$
\Delta_{\alpha \beta}(v)=\left[\chi_{\alpha \beta}(v)-\chi_{\beta \alpha}(-v)\right] \mathcal{T}_{\beta \gamma}-2 i v \mathcal{C}_{\alpha \gamma}(v) .
$$

We find that the GFDT (the FDT in the co-moving frame associated with the mean limit cycle $[29,31]$ ) is indeed obeyed for a state-independent drive. When $b^{\prime \prime} \neq 0$, we observe deviations from the predictions of this fluctuation-dissipation theorem proportional to $b^{\prime \prime}$, which we may write in terms of the deviation matrix defined in Eq. (18),

$$
\Delta(v)=\frac{2 b^{\prime \prime}}{v\left(4 \mu^{2}+v^{2}\right)}\left[\begin{array}{cc}
0 & -v+2 i \mu \\
v+2 i \mu & \frac{-i 4 b^{\prime \prime} \mu}{b^{\prime}}
\end{array}\right] .
$$

Only the Lorentzian fluctuations of the radial $\delta r$ variable obey the GFDT when the drive is state dependent. When $b^{\prime \prime} \neq 0$, the feedback between the azimuthal driving force and the radial oscillations breaks the GFDT due to both new cross correlations $C_{r \phi}$ and the modified phase diffusion, seen in $C_{\phi \phi}$. We suspect that the violation of this GFDT due to the state-dependent drive cannot be removed by an appropriate change of observables, as has been explored for nonequilibrium fluctuations about a fixed point $[40,41]$.

In Fig. 4(a), we show the correspondence between the correlation data obtained from numerical simulations (dark blue circles) and that expected from the response function (orange crosses) for the radial variable based on the GFDT.

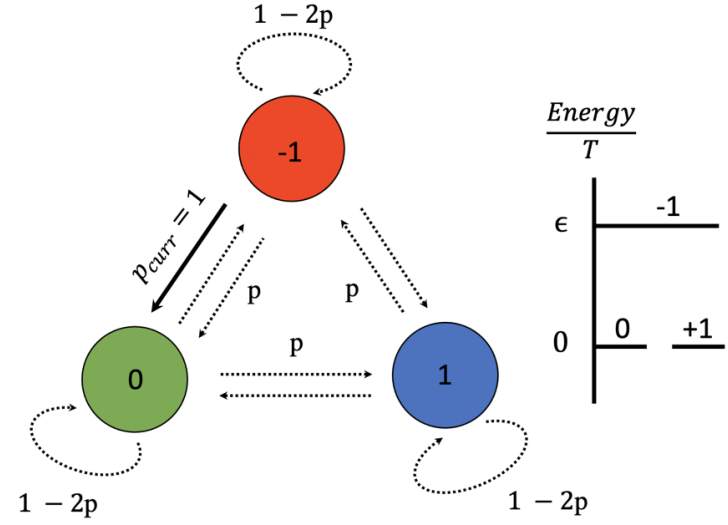

FIG. 5. A schematic diagram of the discrete-time three-state system, showing states $\{-1,0,1\}$ denoted by red, green, and blue disks, respectively. These states have energies $\{\epsilon, 0,0\}$. In the equilibrium steady state, $p_{\text {curr }}=0$. Conversely, when $p_{\text {curr }}=1$ or 2 , the system has a nonzero internal probability current which may be removed by working in a co-moving frame (see Appendix E for details).

In Fig. 4(b), where we compare the frequency-dependent phase diffusion constant measured from the numerical data (red circles) and the GFDT-based prediction (orange crosses), we see the failure of the GFDT for the system driven in a state-dependent manner. Clear deviations are seen at low frequencies, as predicted by Eq. (19). When the drive is independent of the state $\left(b^{\prime \prime}=0\right)$-see Fig. 14 in Appendix D-these deviations vanish and the GFDT is once again obeyed. We show the real and imaginary parts of $\chi_{\phi r}(v)-$ $\chi_{r \phi}(-v)$ in Figs. 4(c) and 4(d), respectively. The former predicts the cross correlations of the radial and phase fluctuations via GFDT. Those predicted orange crosses illustrated in Fig. 4(c) also fail to agree with the simulation data (green circles). In all panels [Figs. 4(a)-4(d)], we show our analytical calculations of $\left[\chi_{\alpha \beta}(v)-\chi_{\beta \alpha}(-v)\right] \mathcal{T}_{\beta \gamma}$ as obtained from Eq. (16) (black lines). These are in universal agreement with the GFDT-based correlation functions obtained from the numerically simulated response function data (orange crosses), which demonstrates the agreement between our analysis of the Hopf model presented in Eqs. (14) and (16) and the numerical simulations.

\section{THREE-STATE MODEL WITH A STATE-DEPENDENT DRIVE}

To better understand the role of a state-dependent drive in breaking GFDT, it is helpful to examine the same phenomenon in a more simple, finite-state model. We analyze two such three-state systems. First, as shown in Fig. 5, we consider a discrete-time system with its three states labeled $s=\{-1,0,+1\}$ and having energies $\{\epsilon, 0,0\}$. When calculating occupation probabilities, the Boltzmann constant $k_{B}$ is assumed to be 1 without loss of generality. The system's dynamics are determined by an internal drive which generates a probability current $p_{\text {curr }}$ taking on a value of 0,1 , or 2 anticlockwise steps per unit time around the triangle of states-see Fig. 5. In addition to this deterministic stepping, we include unbiased stochastic hopping. 
For $p_{\text {curr }}=1$, the occupation probability distribution traverses from state $m$ to $n$ such that $n$ lies circularly to the right of $m$ in the set $\{-1,0,1\}$. When $p_{\text {curr }}=2$, it traverses in the opposite direction. Meanwhile, the hopping rate $p, 0 \leqslant p \leqslant$ 0.5 , is unbiased when $\epsilon=0$ and generates diffusion among the three states. Thus the stochastic system $(p>0)$ with no current $\left(p_{\text {curr }}=0\right)$ obeys detailed balance and corresponds to an equilibrium system.

The three cases we explore in this finite-state model are: detailed balance, an internal drive with constant probability current, and a drive that generates a state-dependent current. We describe the systems with a nonzero current in further detail here. We also refer the reader to Appendix E, in particular Table I, for the complete set of transition probabilities defining the system. To quantify the work done by the internal drive that generates a constant $p_{\text {curr }}=1$, we consider a simple detailed-balance argument with $\epsilon=0$ and compute the transition probabilities between states -1 and 0 . If detailed balance were to hold, the asymmetry in those probabilities introduced by the current implies that there is an energy difference between these states, $\Delta \varepsilon=\varepsilon_{0}-\varepsilon_{-1}=\ln [p /(1-2 p)]$ (when measured in units of $k_{\mathrm{B}} T$ ). The work performed by the drive on the system is then given by the product of $\Delta \varepsilon$ and the difference in the forward and backward hopping probabilities between the states 0 and -1 . We emphasize that the work done is not equivalent to introducing a time-independent potential into the three-state system. Rather, one may visualize the drive as changing the triangle representing the three states into a helix that continually descends in energy as one traverses the cycle in the anticlockwise direction. To make contact with the three-state model, one must also associate each state on the helix with the one vertically above-see Fig. 19 in Appendix E for a schematic representation.

We incorporate state dependence in the drive by allowing the value of $p_{\text {curr }}$ to change in response to the history of state occupation, which allows us to study the finite-state analog of the Hopf oscillator with a state-dependent drive $\left(\omega_{0}=0, b^{\prime \prime} \neq\right.$ $0)$. We incorporate this by setting

$$
p_{\text {curr }}\left(t_{i}\right)=\left\lfloor\sum_{j=1}^{\infty} r e^{\lambda(i-j)} \xi\left(t_{i-j}\right)\right\rfloor \bmod 3,
$$

where $\lfloor$.$\rfloor is the floor function returning the integer part of its$ argument. We have also introduced the function $\xi\left(t_{i-j}\right)$, which takes the value $1,-1$, or 0 when the system is in state $1,-1$, or 0 , respectively, at time $t_{i-j}$. In turn, $\xi\left(t_{i}\right)$ is defined using the indicator functions $\sigma_{k}\left(t_{i}\right)$, which are $1(0)$ when the system is (is not) in state $k$ at time $t_{i}$ :

$$
\xi\left(t_{i}\right)=(-1)^{\sigma_{-1}\left(t_{i}\right)}\left[1-\sigma_{0}\left(t_{i}\right)\right] .
$$

Finally we note that the state-dependent current depends on two constants $r$, which determines the responsiveness of its measurement, and $\lambda$, which sets the memory time $\sim \lambda^{-1}$. We first perform numerical simulations of the symmetric model $(\epsilon=0)$ that obeys detailed balance $\left(p_{\text {curr }}=0\right)$. We tracked the stochastic trajectories $(p=0.02)$ of 40 realizations of the system over a total of $4 \times 10^{4}$ time steps for each of the realizations. Setting $\epsilon=0$ resulted in the occupation probability of the three states being one-third, as expected (not shown). From these trajectories, we also compute all two-point corre-

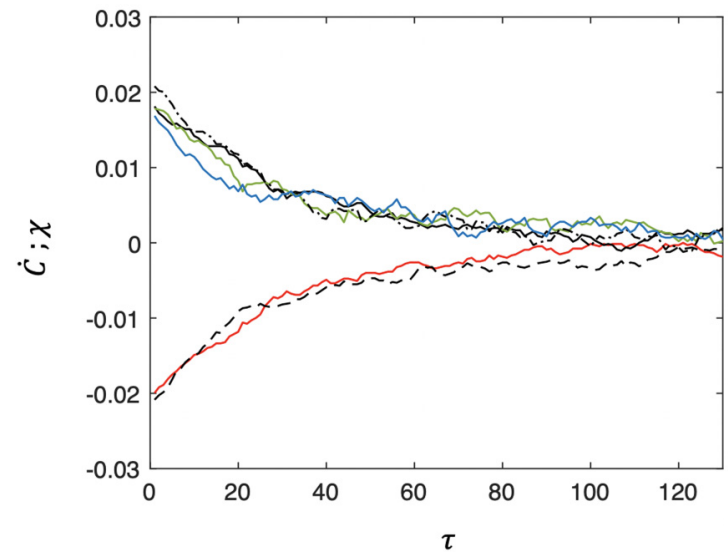

FIG. 6. Test of FDT for the equilibrium system. By comparing $\dot{C}_{-1,-1}$ (black dashed line) and $\chi_{-1,-1}$ (red line), we check that the response of the system to a force driving it out of the -1 state matches the appropriate correlation function derivative. We also find the expected correspondence between $\dot{C}_{0,-1}$ (black solid line) and $\chi_{0,-1}$ (green line) as well as $\dot{C}_{1,-1}$ (black dash-dotted line) and $\chi_{1,-1}$ (blue line).

lation functions,

$$
C_{n m}(\tau)=\frac{1}{2}\left[\left\langle\sigma_{n}\left(t_{i}+\tau\right) \sigma_{m}\left(t_{i}\right)\right\rangle+\left\langle\sigma_{m}\left(t_{i}+\tau\right) \sigma_{n}\left(t_{i}\right)\right\rangle\right] .
$$

The average is taken over an ensemble of trajectories at time delay $\tau$. Under the assumption of ergodicity, one may alternatively average over longer time series from one trajectory. Further, an experimentalist investigating the stochastic dynamics of a nonequilibrium steady-state system might implicitly assume time-reversal invariance. Therefore, our definition of the correlation function was chosen to make it explicitly time-reversal invariant when $n \neq m$. Clearly, if the driven system admits a nonvanishing probability current, this symmetry will not be valid. However, since we propose using the violation of fluctuation-dissipation theorems as a test for both an underlying limit cycle in general and one driven in a state-dependent manner in particular, we will suppose a priori that the correlation data is analyzed assuming time-reversal invariance in the steady state.

To test the standard FDT, we numerically obtained the response of the occupation probability of state $n, p_{n}(t)=$ $\left\langle\sigma_{n}(t)\right\rangle$, to a force conjugate to the occupation of state -1 ,

$$
\delta p_{n}\left(t_{i}\right)=-\sum_{j=-\infty}^{i} \chi_{n,-1}\left(t_{i}-t_{j}\right) \delta \epsilon_{-1}\left(t_{j}\right),
$$

by setting the energy of that state to $\epsilon_{-1}=3$ for one time step and observing the subsequent stochastic evolution of the system. We confirmed that our perturbation was in the linear response regime by varying $\epsilon_{-1}$; see Appendix F. In Fig. 6, we plot $\chi_{-1,-1}(\tau), \chi_{0,-1}(\tau)$, and $\chi_{1,-1}(\tau)$ as solid red, green, and blue curves, respectively. As expected, the transient increase in the energy of the -1 state suppresses the occupation probability of that state and symmetrically increases the occupation probability of the other two states: +1 and 0 . The system recovers its equilibrium probabilities exponentially with a decay rate of about 20 inverse time units. 


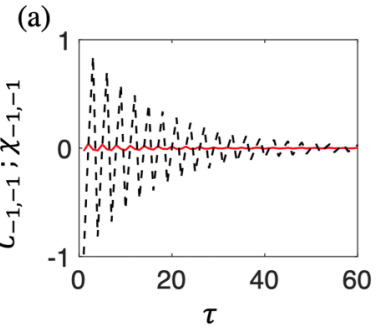

(b)

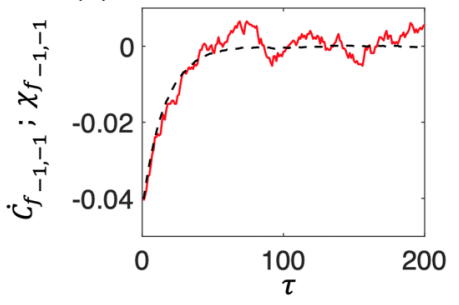

FIG. 7. (a) In the presence of a constant nonzero internal probability current, the system violates the FDT, as can be seen in the comparison of the linear response function $\chi_{-1,-1}$ (red) and the time derivative of its autocorrelation function $\dot{C}_{-1,-1}$ (black dashed). (b) However, upon transforming to the co-moving reference frame, we show that the three-state model satisfies GFDT. The derivative $\dot{C}_{f-1,-1}$ (dashed black) and the response function $\chi_{f-1,-1}$ (red) are now in agreement.

The standard FDT requires these response functions to be equal to the time derivative of the correlation functions $\dot{C}_{n,-1}(\tau)$ evaluated at time delay $\tau$. We plot the numerically obtained time derivatives of the correlation functions $\dot{C}_{-1,-1}$, $\dot{C}_{0,-1}$, and $\dot{C}_{1,-1}$ as dashed black, solid black, and dash-dotted black lines, respectively, in Fig. 6. As expected, we find that the time derivatives of the correlation functions of state occupation agree with the responses of the occupation probability to a force conjugate to that variable. All the correlation functions in this figure were normalized such that $C_{n, m}(0)=\delta_{n, m}$. The response functions were multiplied by temperature, in this case of value 0.2 . The remaining plots for this system are all normalized using this value.

We now consider the case of an internal drive generating a constant nonzero probability current, setting $p_{\text {curr }}=1$. In Fig. 7(a), we demonstrate the violation of FDT. The red curve is the numerically computed response function $\chi_{-1,-1}(\tau)$, and the dashed black curve is the derivative of the corresponding correlation function $\dot{C}_{-1,-1}(\tau)$, whose oscillatory nature can be attributed to the probability current in the model. The standard FDT requires these to be equal. They are not equal, indicating the breakdown of FDT. However, for this model, we propose that one may obtain a valid GFDT similar to that of Refs. [29,31] by evaluating the correlation and response functions in a reference frame co-moving with velocity $p_{\text {curr }}$. Note that since we model this three-state system after the stochastic, driven Hopf oscillator, we choose the angular velocity of the co-moving frame to be equal to that of the driven deterministic system. To transform to the co-moving frame, we introduce new indicator functions,

$$
\tilde{\sigma}_{i}\left(t_{j}\right)=\sigma_{\left(i+v_{\text {drift }} t_{j}\right) \bmod 3} .
$$

We find that in the co-moving frame, the numerically computed response function of $\chi_{f-1,-1}$ (red curve) agrees with $\dot{C}_{f-1,-1}$ (dashed black line), as seen in Fig. 7(b). The values of these two functions, as a matter of fact, are similar to those of the equilibrium system (Fig. 6). Due to the symmetry of the problem, we only show plots for the -1 state. For the other two states, we refer the reader to Figs. 15 and 16 in Appendix D. (a)

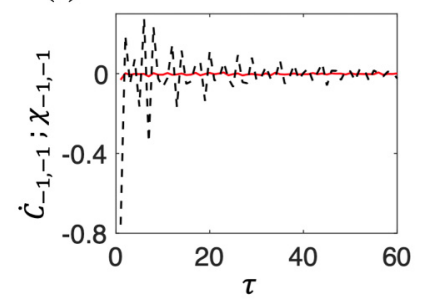

(b)

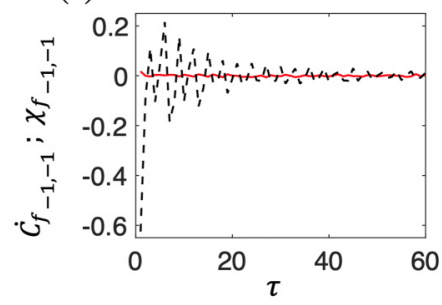

FIG. 8. The three-state model with a state-dependent probability current [see Eq. (20)]. (a) We juxtapose the derivative $\dot{C}_{-1,-1}$ (dashed black) and the response function $\chi_{-1,-1}$ (red) to illustrate the breakdown of FDT. (b) Furthermore, unlike the one illustrated in Fig. 7 , this system also violates GFDT, as is evident by comparing $\dot{C}_{f-1,-1}$ and $\chi_{f-1,-1}$, calculated in the associated co-moving frame.

Next, we study the three-state model with a statedependent drive by choosing $r=2$ and $\lambda=0.1$. This non-Markovian system violates FDT, as shown by the plots in Fig. 8(a). The time derivative of the autocorrelation $C_{-1,-1}$ (black dashed line) deviates appreciably from the response function $\chi_{-1,-1}$ (shown in red). Plots for the other two states are given in Appendix G. Moreover, in the co-moving frame, the state-dependent nature of the probability current precludes restoration of the generalized theorem [Fig. 8(b)]. In order to test the GFDT in the rotating frame, we chose a reference frame co-moving with the average current, which in our simulations was 1 . There exists no other reference frame that may restore the GFDT in this driven system. The breakdown of both FDT and GFDT relations in the system is similar to our observations in the Hopf limit cycle oscillator with a state-dependent drive, i.e., $b^{\prime \prime}>0$.

One may ask whether any time variation of the probability current is sufficient to invalidate the FDT or the GFDT. To address this, we considered a randomly varying drive that generates the same average current as the state-dependent drive. In particular, we consider a three-state model with a randomly varying internal current that has equal probabilities at each time step of being 0,1 , or 2 . There are no temporal correlations in the stochastic $p_{\text {curr }}$. It is easy to see that the mean probability current is unity. This system, unlike the three-state model with a state-dependent drive, obeys the GFDT (data not shown). We conclude that the state-dependent mechanism of the drive is required to invalidate the GFDT.

We also examine the stochastic dynamics of a more general three-state model defined by the continuous-time master equation for the probability $p_{n}(t)$ of observing the system in state $n=-1,0,1$ at time $t_{i}$,

$$
\dot{p}_{n}\left(t_{i}\right)=\sum_{m \neq n}\left[p_{m}\left(t_{i}\right) \alpha_{m n}\left(t_{i}\right)-p_{n}\left(t_{i}\right) \alpha_{n m}\left(t_{i}\right)\right] .
$$

See Fig. 9 for a schematic representation. The system evolves via six transition rates, e.g., the transition rate from state $n$ to $m$ at time $t_{i}: \alpha_{n m}\left(t_{i}\right)$. These six transition rates are given by the following rules. We set

$$
\alpha_{n m}\left(t_{i}\right)=\alpha_{m n} e^{\epsilon_{n}-\epsilon_{m}} \alpha\left(t_{i}\right),
$$

where $n$ is to the right of $m$ in the list of states $\{-1,0,1\}$ or its cyclic permutations. Transition probabilities are then 

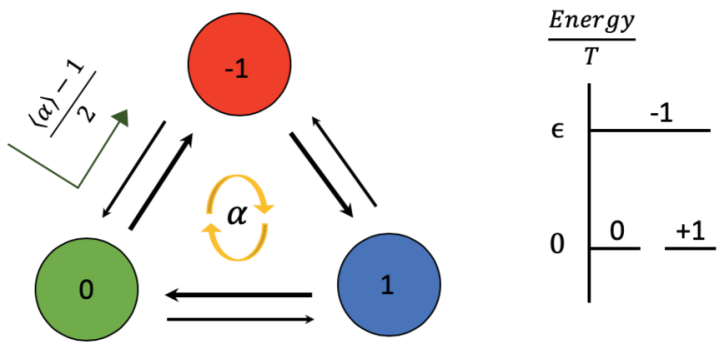

FIG. 9. A schematic diagram of the continuous-time three-state system, showing states $\{-1,0,1\}$ denoted by red, green, and blue disks, respectively. These states have energies $\{\epsilon, 0,0\}$. In the nonequilibrium steady state, the clockwise transition rates are enhanced over their detailed-balance values by $\alpha(t)$. The resulting steady-state nonzero probability current may again be removed by working in a co-moving frame.

the product of the respective rates and a simulation time step duration of $10^{-2}$. The bare rates $\alpha_{m n}=\alpha_{n m}$ (both set to one in our simulations) provide for the undriven system maintaining detailed balance. Meanwhile, the factor $\alpha(t)$ breaks detailed balance as is required by the drive. Thus the strength of the drive is now modulated by $\alpha\left(t_{i}\right)$, which allows the drive to depend at time step $t_{i}$ on the history of prior state occupation, as shown in Eq. (27). By choosing $\alpha(t)$ to be a constant greater than one, we generate a clockwise probability current (see Fig. 9) in the steady state. Such a choice is analogous to turning on a state-independent drive in the isochronous Hopf model $\left(\omega_{0} \neq 0, b^{\prime \prime}=0\right)$ of the hair cell oscillator. Later, to introduce a state-dependent process, we will consider the case in which the probability current depends upon the history of the system by setting

$$
\alpha\left(t_{i}\right)=1+r \sum_{j=1}^{\infty} e^{\lambda(i-j)} \xi\left(t_{i-j}\right),
$$

where $\xi\left(t_{i-j}\right)$ has been defined in Eq. (21).

The strength of the dependence is again controlled by $r . \lambda$ controls the exponential decay rate of the memory kernel in Eq. (27). It is measured in inverse time units $\delta t=t_{i+1}-t_{i}$, which we always set to 0.01 . The effect of the feedback is to increase the probability current when the system has recently been in the +1 state and decrease it when the system has visited the -1 state. The simulations for this three-state model, as for the one before, were performed using 40 realizations over $4 \times 10^{4}$ time steps. For additional details of these simulations, we refer the reader to Appendix A.

We first study the detailed-balance system, which can easily be shown to be equivalent to the nondriven case of the first three-state model. In Fig. 10, we illustrate the time derivatives $\dot{C}_{-1,-1}(\tau), \dot{C}_{0,-1}(\tau)$, and $\dot{C}_{1,-1}(\tau)$ as the dashed black, solid black, and dot-dashed black lines, respectively. Also shown are the linear response functions of $\chi_{-1,-1}(\tau)$ (red), $\chi_{0,-1}(\tau)$ (green), and $\chi_{1,-1}(\tau)$ (blue), which, as anticipated, overlap with their corresponding correlation derivatives. The temperature of this system is 0.11 . We now repeat this measurement in a nonequilibrium system by setting $\alpha(t)=98$. The resulting drive breaks detailed balance by generating a state-independent probability current and is similar to the Hopf model of hair cell oscillations with $b^{\prime \prime}=0$ but $\omega>0$. In

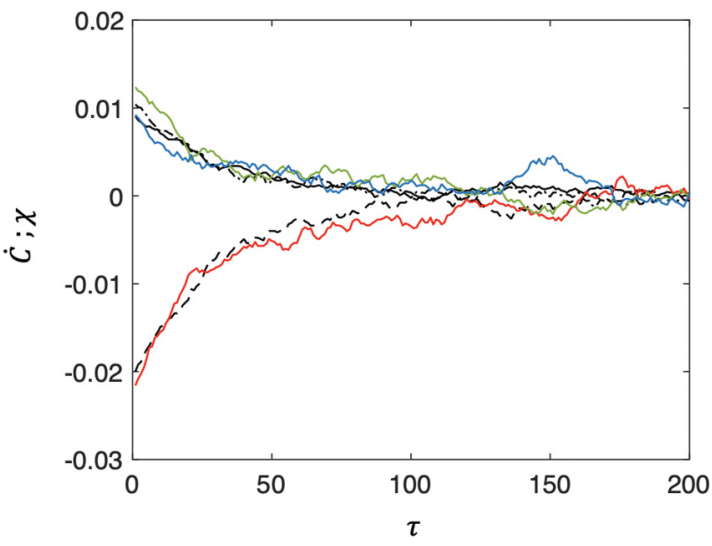

FIG. 10. The FDT relation is satisfied by the detailed-balance system. As in Fig. 6, we compare the response of the system to a change in the energy of state -1 with the corresponding correlation function derivatives. We show $\dot{C}_{-1,-, 1}$ (dashed black), $\dot{C}_{0,-, 1}$ (solid black), $\dot{C}_{1,-, 1}$ (dot-dashed black), $\chi_{-1,-, 1}$ (red), $\chi_{0,-, 1}$ (green), and $\chi_{1,-, 1}$ (blue).

Fig. 11(a), we show the measured response function $\chi_{1,-1}(\tau)$. As in the equilibrium case, the applied force pushes the system out of the -1 state into the 0,1 states. But, unlike the equilibrium case, the change in probability oscillates in time due to the detailed-balance-breaking drive. For example, the occupation probability of +1 cycles the three-state system in the clockwise direction, while slowly decaying over longer times (not shown), resulting in an oscillatory response function as in the solid blue line in the figure. The correlation function $C_{1,-1}(\tau)$ also shows this oscillatory behavior, but its derivative (dashed black line) does not match the corresponding response function. The standard FDT is violated.

We can, however, obtain a GFDT in the driven system by working in a "rotating" reference frame-one that moves with the constant clockwise probability current of the nonequilibrium steady state. We derive the frame's velocity using the master equation in Appendix H. Moving at the speed of $\frac{\alpha-1}{2}$, we now find that the response function of the +1 state in this co-rotating frame to a force acting on the -1 state- the dashed blue line in Fig. 11(b)—agrees with the numerically

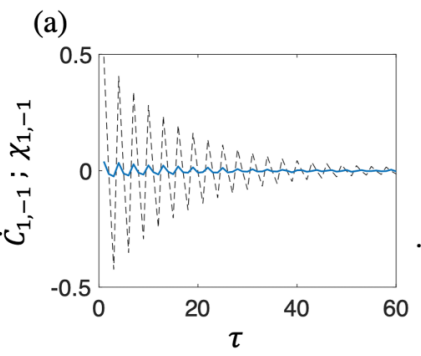

(b)

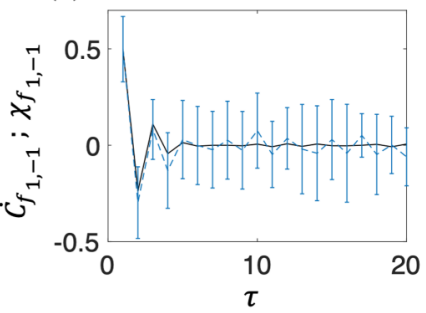

FIG. 11. (a) FDT violation in the broken detailed-balance system at $\alpha(t)=98$. We compare the time derivative of the cross-correlation $\dot{C}_{1,-1}$ (dashed black line) and the response $\chi_{1,-1}$ (blue line) functions. (b) In the co-moving frame, the GFDT holds, as seen by comparing the cross-correlation $\dot{C}_{f_{1,-1}}$ (black line) and response $\chi_{f_{1,-1}}$ (dashed blue line) functions. Error bars denote the standard deviation of the mean. 

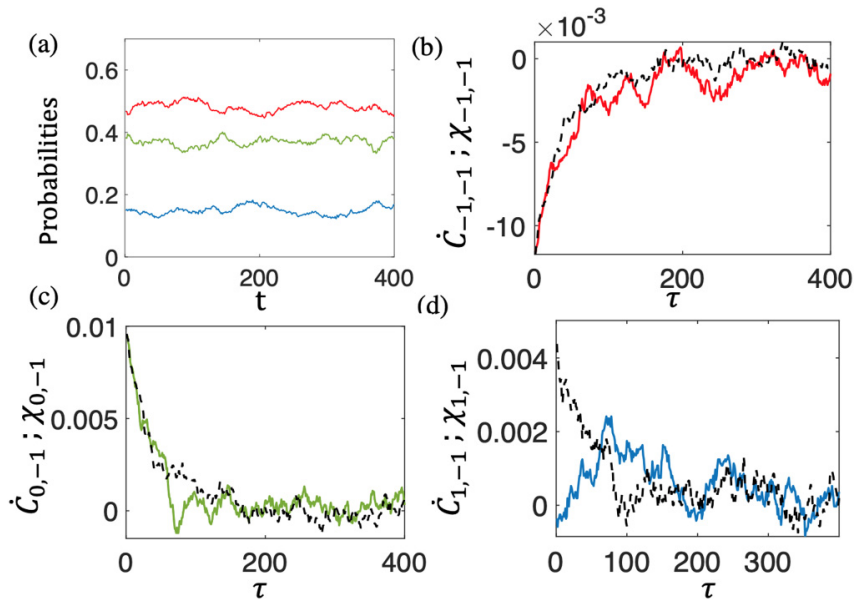

FIG. 12. The three-state system with a state-dependent drive, given by Eq. (27). (a) With the drive parameters at $r=0.095$ and $\lambda=0.1$, the states are no longer occupied with equal probability, even when their occupation energies are equal. (b)-(d) Comparison of $\dot{C}$ (black dashed) with the appropriate $\chi$ (color solid) in the nonrotating lab frame. In (d), we observe significant deviations from the FDT.

measured time derivative of the correlation function [calculated using Eq. (22)], shown as the black line in this figure. The error bars represent the standard deviation of the mean for the response data. We find a similar agreement between the other correlation and response functions in the co-rotating frame; these are shown in Appendix D (Figs. 17 and 18). While neither the time derivative of the correlation function nor the response function in a system with a constant internal probability current agrees with predictions based on the equilibrium system, their agreement with each other shows that a generalized fluctuation-dissipation theorem holds in such a system, as expected based on the work of Seifert and collaborators $[29,31]$. The appearance of the GFDT in the co-rotating frame, which zeros out the steady-state probability current, is analogous to our observation of a similar fluctuation-dissipation theorem in the isochronous Hopf oscillator system.

We now introduce a drive that generates state-dependent probability current in this more general three-state system via Eq. (27), taking $r=0.095$ and $\lambda=0.1$. This is analogous to the nonisochronous Hopf system in that the drive now depends on the state of the system. By analogy, we expect to observe the breakdown of the GFDT once again. With this state-dependent drive, we obtain a steady-state system with nonequal occupation probabilities of the three states in a steady state. In spite of the fact that the energies of all three states are equal, the drive breaks the permutation symmetry of these states, as shown in Fig. 12(a). As a result, the simple occupation probabilities of the states in this nonequilibrium steady state do not reflect their relative energies. Conversely, just by observing these occupation probabilities, one might conclude erroneously that this system was in equilibrium with a particular spectrum of energy levels. To test this conclusion, one must not only examine these probabilities, but also compare the correlation and response functions of the system.
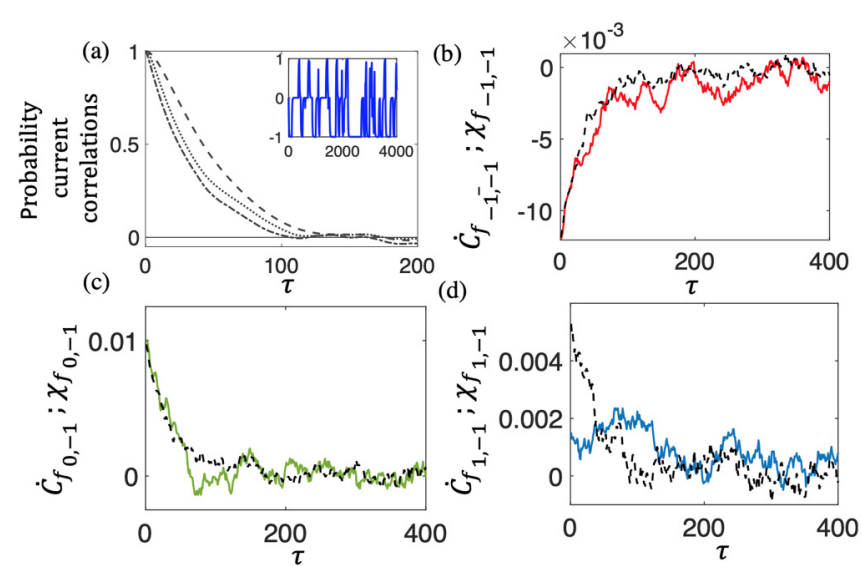

FIG. 13. (a) Correlation of the steady-state probability current $\alpha(t)-1$ for different $\lambda$ and $r$ values: $r=0.095, \lambda=0.1$ (dashed line), $r=0.095, \lambda=0.25$ (dotted line), $r=0.095, \lambda=1$ (dotdashed line), and $r=0$ (solid line). For $r=0.095$ and $\lambda=0.1$, we show the stochastic current from a representative trajectory (inset). (b)-(d) Illustration of the violation of the GFDT when working in a co-moving frame that works to eliminate the mean probability current. Comparing with Fig. 12, we see that this frame partially, but rather imperfectly, restores the fluctuation-dissipation theorem.

In Figs. 12(b)-12(d), we show a comparison of the time derivative of the correlation function $\dot{C}_{k,-1}(\tau)$ and the response function $\chi_{k,-1}(\tau)$ for $k=-1,0,+1$, respectively. The standard FDT fails dramatically for one set of measurements: $\dot{C}_{1,-1}(\tau) \neq \chi_{1,-1}(\tau)$-see Fig. 12(d).

We examine the probability current in this more general continuous-time three-state model. We show its temporal correlation function in a system with a state-dependent current in Fig. 13(a). In the inset of the same panel, we show a representative part of the time series of the probability current from which the correlation functions were obtained. Clearly, as $\lambda$ is decreased (drive memory time increased), the probability current's correlation time increases, so that the effect of the drive's state dependence is reduced. Its current value depends on a long time average of the system, which itself necessarily varies only slowly in time. As a result, we find that with sufficiently long memory times, the system whose drive is state dependent begins to resemble one whose drive is uncorrelated with the state, as long as $r / \lambda$ remains fixed. As a result, the magnitude of the violations of the GFDT will decrease.

Given this intuition, it is interesting to examine the residual violation of the GFDT in a system driven by a weakly state-dependent drive. Due to the current fluctuations that are still correlated with the state of the system, it is clear that no co-moving frame can precisely reestablish the GFDT. But we can find the best approximation to the GFDT in this system by working in a co-moving reference frame selected to eliminate the mean probability current, i.e., we chose a velocity $\frac{\langle\alpha\rangle-1}{2}$ to minimize GFDT discrepancies. In Figs. 13(b)-13(d), we do this. The results shown in Figs. 13(c) and 13(d) demonstrate that the GFDT still fails due to feedback between the system and the drive. But a comparison between Fig. 13(c) and Fig. 13(d) measured in the co-moving frame with Fig. 12(c) and Fig. 12(d), showing the same quantities in the nonrotating laboratory frame, demonstrates that GFDT may be partially 
restored. This restoration can be quantified using the $L 2$ norm of $\dot{C}_{-1, m}-T \chi_{-1, m}$ for state $m$, whose value is $2.5 \times$ $10^{-5}, 4 \times 10^{-5}$, and $10^{-4}$, respectively, when $m=-1,0$, and 1. Notably, any restoration of GFDT is visible only at short timescales of $\tau<40$ when the memory time of the drive is $\lambda=0.1$. With even weaker state dependence of the drive, this restoration of the GFDT further improves (data not shown), demonstrating that the magnitude of the GFDT deviations can be used as a proxy for assessing both the integration time of the drive and its responsiveness, defined as the degree to which its energy input varies in response to the state of the system.

\section{SUMMARY}

Systems that exhibit nonequilibrium steady states frequently violate the FDT. Failure to satisfy the conditions set by that theorem has therefore been used as a test of the nonequilibrium nature of various stochastic steady states, indicating the presence of an energy-consuming process. As complex biological systems invariably contain active processes, such a test is useful for experimentally quantifying precisely which degrees of freedom in the system are out of equilibrium and thereby learning something about the underlying processes maintaining that nonequilibrium steady state. For example, in actomyosin gels, one observes enhanced strain fluctuations at low frequencies due to motor activity. This is a consequence of the fact that the motor dynamics introduce force autocorrelations with a colored noise spectrum. As a result, the strain fluctuations observed across multiple timescales do not correspond to the equilibrium (visco)elastic system at any single temperature. In this and other systems, the quantitative measurement of the breakdown of the FDT is a type of sensor for the detection of nonequilibrium steady states and for providing a measure of how far from equilibrium they are.

Broadly speaking, there are multiple ways to violate the FDT, and, as a consequence, not all FDT violations have the same implications. A large class of nonequilibrium biological systems breaks detailed balance by having nonvanishing probability currents in the steady state. We have explored one particular class of such systems: those exhibiting stochastic limit cycles. In this case, previous work by Seifert et al. has introduced a new type of generalized fluctuation-dissipation theorems, i.e., GFDTs, based on working in a co-moving reference frame that effectively eliminates the stationary probability current. When fluctuations are viewed in this comoving reference frame, the familiar relations between them and the response functions of the system are restored.

In the current work, we introduce a different feature: the internal drive maintaining the limit cycle, in effect, measures the state of the system and modifies its power generated based on that measurement. In systems driven by such a state-dependent drive, we demonstrated the violation of the Seifert-Speck GFDT. We first examined the Hopf oscillator model with a state-dependent drive, where the quantitative degree of GFDT violation is proportional to a single model parameter $b^{\prime \prime}$, which measures the ability of the azimuthal drive to modulate the oscillator's power input in response to the radial excursions of the system.
To isolate the role of state dependence of a drive in breaking the GFDT in an even simpler model, we introduced two related three-state systems-one described using discretetime dynamics and the other being continuous time in nature. In both of these, we violate detailed balance by producing stationary states with a nonzero probability current. These systems then violated the standard FDT, as expected. By introducing a co-rotating frame to eliminate the probability current in the three-state system with a state-independent drive, we obtain a GFDT, which is consistent with previous work [29,31]. But when we allow the probability current to change based on the history of the system's trajectory, we once again observe the breakdown of the GFDT. We also observed that the feedback between the drive and the state of the system is crucial for GFDT violations. These vanish if the power generated by the drive varies randomly in time in a manner uncorrelated with the state of the system. These results are in direct analogy with the more complex Hopf model and allow us to more carefully probe the role of a state-dependent drive in the violation of the GFDT.

The Hopf model is, in fact, the simplest model for describing the dynamics of hair cell motion. As such, it provides an important connection between the basic questions of fluctuation-dissipation theorems (or their failure) in driven steady states with underlying state-dependent drives and stochastic dynamics in a living system. It also presents us with a relatively simple biological dynamical system in which to experimentally explore fluctuation-dissipation theorems in nonequilibrium steady states. Our previous work looking at hair cell fluctuations in the Frenet frame co-moving with the mean probability current of the system generated correlation data consistent with the theory discussed here [24]. Future work is needed to examine the response functions of the system in order to test the GFDT.

Based on our current work, we propose that just as the failure of the FDT has been used to test for nonequilibrium steady states, one should be able to look for the breakdown of the GFDT as a test of stochastic steady states driven out of equilibrium by a state-dependent drive. Two emblematic features of living systems are long-lived nonequilibrium steady states and homeostasis. One method to maintain homeostatic control of driven states is through a drive that measures and consequently modulates its activity based on the current state of the system, as seen in the nonisochronous hair cell model. As many biological systems may contain homeostatic control that is not as readily accessible experimentally, we suggest that the breakdown of the GFDT may serve as a useful tool to indicate the presence of and to quantify the efficacy of such feedback-based control. One experimentally tractable biological system in which one might test the correlation and causation between an internal state-dependent drive and violation of GFDT is the spontaneously oscillating hair cell of the inner ear.

\section{ACKNOWLEDGMENTS}

A.J.L. acknowledges partial support from Grant No. NSFDMR-1709785. D.B. acknowledges partial support from the NSF Physics of Living Systems, under Grant No. 1705139. 
J.S. acknowledges partial support from a Fletcher Jones Foundation fellowship.

\section{APPENDIX A: SIMULATION DETAILS}

\section{A. Hopf oscillator}

The stochastic and externally perturbed Hopf oscillator of Eq. (1) was simulated using the fourth-order Runge-Kutta method for a duration of $60 \mathrm{~s}$, with a time step of $10^{-4} \mathrm{~s}$. We explore a large range in the amplitude of the noise variances $\left\langle\eta_{x}^{2}\right\rangle$ and $\left\langle\eta_{y}^{2}\right\rangle$ (where $\left\langle\eta_{x}^{2}\right\rangle=\left\langle\eta_{y}^{2}\right\rangle$ ) covering $10^{-7}$ to 0.4 , as well as a range of perturbative forces $10^{-3}$ to $10^{-1}$. All throughout, the amplitude of mean limit cycle oscillators was held to be $O(1)$. While consistent results were obtained over the full span of these values, Figs. 3 and 4 employ the highest value of force and noise in their respective ranges.

\section{B. Mean limit cycle of the Hopf oscillator}

The Hopf oscillator's phase space $\{-\pi, \pi\}$ is partitioned into 200 bins. Trajectories in each bin are then averaged, resulting in the mean curve.

\section{Three-state model}

Equation (25) was numerically computed using a randomnumber generator that outputs a value in the range [0-1]. Comparison of this value with the occupation probabilities of the three states determines the stochastic trajectory for each of the 40 realizations. Data were always taken after running the system long enough so that its initial conditions were no longer relevant. All simulations were performed using MATLAB [42].

\section{APPENDIX B: ELECTRICALLY CHARGED PARTICLE IN A MAGNETIC FIELD}

The motion of a damped, harmonically bound charged particle of mass $m$ and charge $e$ in the $x y$ plane under the influence of magnetic field $H \hat{z}$ is given by

$$
\ddot{\hat{r}}+\gamma \dot{\hat{r}}+\omega_{0}^{2} \hat{r}=\frac{e}{m c} \dot{\hat{r}} \times H,
$$

where $\gamma$ is the friction coefficient, $\omega_{0}$ is the natural frequency of the oscillator $\left(\omega_{0}=\sqrt{k / m}\right.$ for a Hookean spring constant $k$ ), and $c$ is the speed of light. The equations of motion may be written in terms of $x$ and $y$ as

$$
\begin{gathered}
\ddot{x}+\gamma \dot{x}+\omega_{0}^{2} x=\frac{e H}{m c} \dot{y}, \\
\ddot{y}+\gamma \dot{y}+\omega_{0}^{2} y=-\frac{e H}{m c} \dot{x},
\end{gathered}
$$

with introduction of the classical Larmor frequency $\omega_{r}=\frac{e H}{m c}$. Upon driving Eqs. (B2) and (B3) using either stochastic or deterministic (externally applied) forces, we obtain

$$
\begin{aligned}
{\left[\begin{array}{l}
x \\
y
\end{array}\right]=} & \frac{1}{\left(-\omega^{2}+\omega_{0}^{2}-i \omega \gamma\right)^{2}-\omega_{r}^{2} \omega^{2}} \\
& \times\left[\begin{array}{cc}
-\omega^{2}+\omega_{0}^{2}-i \omega \gamma & -i \omega_{r} \omega \\
i \omega_{r} \omega & -\omega^{2}+\omega_{0}^{2}-i \omega \gamma
\end{array}\right]\left[\begin{array}{l}
\eta_{x} \\
\eta_{y}
\end{array}\right] .
\end{aligned}
$$

When considering these as Langevin equations, we assume rotationally symmetric thermal noise so that $\left\langle\eta_{x}^{2}\right\rangle=\left\langle\eta_{y}^{2}\right\rangle=$ $\left\langle\eta^{2}\right\rangle$.

Since the dynamics in the directions $\hat{x}$ and $\hat{y}$ are symmetric, we compute and compare one of each of the autocorrelation and cross-correlation functions. A lengthy but straightforward calculation yields the following response and correlation functions. In order to confirm the validity of the FDT, we present the response functions in combinations such that these combinations should be equivalent to the derivatives of the corresponding correlation functions. We find

$$
\begin{gathered}
\frac{\tilde{\chi}_{x x}(\omega)-\tilde{\chi}_{x x}(-\omega)}{2 i}=\frac{\gamma \omega\left[\left(\omega_{0}^{2}-\omega^{2}\right)^{2}+\gamma^{2} \omega^{2}+\omega_{r}^{2} \omega^{2}\right]}{\left[\omega^{2} \gamma^{2}+\left(\omega_{0}^{2}-\omega^{2}-\omega \omega_{r}\right)^{2}\right]\left[\omega^{2} \gamma^{2}+\left(\omega_{0}^{2}-\omega^{2}+\omega \omega_{r}\right)^{2}\right]}, \\
C_{x x}=\frac{\left\langle\eta^{2}\right\rangle\left[\left(\omega_{0}^{2}-\omega^{2}\right)^{2}+\gamma^{2} \omega^{2}+\omega_{r}^{2} \omega^{2}\right]}{\left[\omega^{2} \gamma^{2}+\left(\omega_{0}^{2}-\omega^{2}-\omega \omega_{r}\right)^{2}\right]\left[\omega^{2} \gamma^{2}+\left(\omega_{0}^{2}-\omega^{2}+\omega \omega_{r}\right)^{2}\right]},
\end{gathered}
$$

and

$$
\begin{gathered}
\frac{\tilde{\chi}_{x y}(\omega)-\tilde{\chi}_{y x}(-\omega)}{2 i}=\frac{\gamma \omega\left(2 i \omega^{3} \omega_{r}-2 i \omega_{0}^{2} \omega \omega_{r}^{2}\right)}{\left[\omega^{2} \gamma^{2}+\left(\omega_{0}^{2}-\omega^{2}-\omega \omega_{r}\right)^{2}\right]\left[\omega^{2} \gamma^{2}+\left(\omega_{0}^{2}-\omega^{2}+\omega \omega_{r}\right)^{2}\right]}, \\
C_{x y}=\frac{\left\langle\eta^{2}\right\rangle\left(2 i \omega^{3} \omega_{r}-2 i \omega \omega_{r} \omega_{0}^{2}\right)}{\left[\omega^{2} \gamma^{2}+\left(\omega_{0}^{2}-\omega^{2}-\omega \omega_{r}\right)^{2}\right]\left[\omega^{2} \gamma^{2}+\left(\omega_{0}^{2}-\omega^{2}+\omega \omega_{r}\right)^{2}\right]} .
\end{gathered}
$$

By direct comparison of Eqs. (B5) and (B6), as well as the cross correlations given by Eqs. (B7) and (B8), we verify that FDT is satisfied for a system responding to a magnetic field. Even though the force is generated from the curl of a vector potential (like our drive in the Hopf system), the magnetic field does not invalidate the FDT since the magnetic forces cannot do work on the system.

\section{APPENDIX C: FOKKER-PLANCK FORMALISM FOR THE HOPF OSCILLATOR}

The time-dependent probability density $p(r, \phi, t)$ of the stochastic Hopf oscillator defined in Eq. (1) evolves as fol- 
lows:

$$
\begin{aligned}
& \frac{\partial p(r, \phi, t)}{\partial t} \\
& =-\frac{\partial}{\partial r}\left[\left(\mu r-b^{\prime} r^{3}\right) p(r, \phi, t)\right]-\frac{\partial}{\partial \phi}\left[\left(\omega+b^{\prime \prime} r^{2}\right) p(r, \phi, t)\right] \\
& \quad+\frac{1}{2}\left\langle\left|\eta_{r}(t)\right|^{2}\right\rangle \frac{\partial^{2}}{\partial r^{2}} p(r, \phi, t)+\frac{1}{2}\left\langle\left|\eta_{\phi}(t)\right|^{2}\right\rangle \frac{\partial^{2}}{\partial \phi^{2}} p(r, \phi, t),
\end{aligned}
$$

where the effective diffusion coefficients $\left\langle\left|\eta_{r}(t)\right|^{2}\right\rangle,\left\langle\left|\eta_{\phi}(t)\right|^{2}\right\rangle$ are constant and are given by Eqs. (11) and (12). We see that when $b^{\prime \prime}=0$, the joint probability distribution for the radial and angular variables decouples so that the joint distribution may be written as a product, $p(r, \phi, t)=p(r, t) p(\phi, t)$. As a result, there are no cross correlations between the radial and angular fluctuations in the system with a state-independent drive. In that case, we obtain

$$
\begin{gathered}
\frac{\partial p(r, t)}{\partial t}=\frac{\partial}{\partial r}\left(-\mu r+b^{\prime} r^{3}\right) p(r, t)+\frac{1}{2}\left\langle\left|\eta_{r}(t)\right|^{2}\right\rangle \frac{\partial^{2}}{\partial r^{2}} p(r, t), \\
\frac{\partial p(\phi, t)}{\partial t}=-\omega \frac{\partial}{\partial \phi} p(\phi, t)+\frac{1}{2}\left\langle\left|\eta_{\phi}(t)\right|^{2}\right\rangle \frac{\partial^{2}}{\partial \phi^{2}} p(\phi, t) .
\end{gathered}
$$

The Hopf oscillator is bounded in the radial direction but has a nonzero, phase-independent probability current in the tangential direction. Writing $r(t)=R_{0}+\delta r(t)$ and expanding about the limit cycle $\delta r(t) \ll R_{0}$, we obtain the steady-state probability currents,

$$
\begin{gathered}
j_{r}=0 \Rightarrow p(r, t) \propto e^{\left[-\frac{2}{\left\langle\left|\eta_{r}(t)\right|^{2}\right\rangle} \mu \delta r^{2}+O\left(\delta r^{4}\right)\right]}, \\
j_{\phi}=\omega p(\phi, t),
\end{gathered}
$$

where the total probability current is $\vec{j}=\hat{e}_{r} j_{r}+\hat{e}_{\phi} j_{\phi}$. We note that the local mean velocity $v(t)$ of the stochastic isochronous Hopf oscillator is given by its deterministic azimuthal current, which is a constant, $\omega$. This allows for the admission of the Seifert-Speck GFDT in the locally comoving Frenet-Serret frame (whose angular velocity is $\omega$ ) associated with such a system (Fig. 14).

However, in the case of the stochastic, nonisochronous Hopf oscillator, the radial and angular fluctuations are correlated, making the joint probability distribution analytically intractable. Therefore, we simply follow from the isochronous Hopf case- the Frenet-Serret frame associated with this oscillator rotates with a constant angular velocity $\omega_{0}$ where, $\omega_{0}=\omega+b^{\prime \prime} R_{0}^{2}$ is the local mean velocity of the deterministic nonisochronous Hopf system. In Fig. 4, we show that this frame is, however, insufficient for the admittance of the GFDT.

\section{APPENDIX D: SYSTEMS WITH A STATE-INDEPENDENT DRIVE}

In the main text, we present three representative systems that incorporate a state-dependent drive. For completeness, (a)
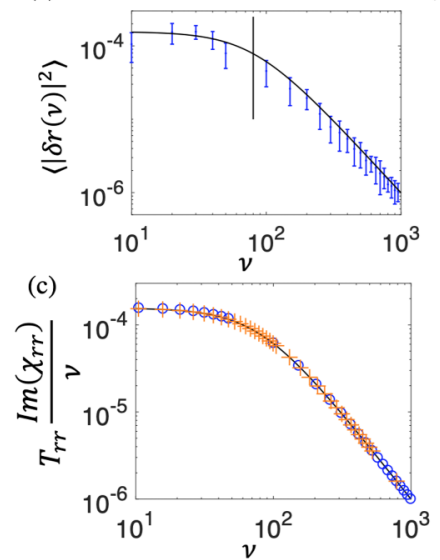

(b)
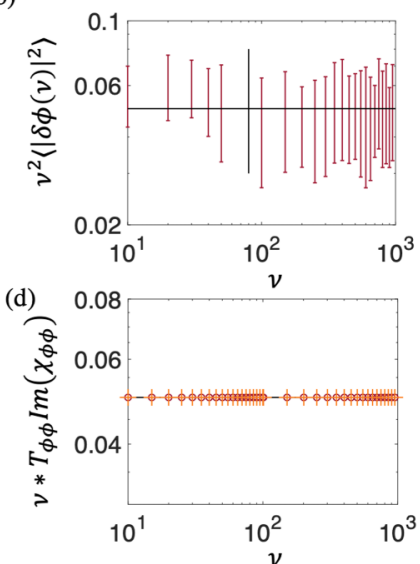

FIG. 14. Correlation and response functions for the isochronous Hopf oscillator $\left(b^{\prime \prime}=0\right)$. (a) Power spectral density of radial fluctuations as a function of frequency $v$ (blue dots). (b) Phase diffusion constant, obtained from the product of the phase fluctuation power spectral density and $v^{2}$ (red dots). In both panels, the vertical (black) line indicates the corner frequency of $2 \mu$. (c),(d) Comparison of the measured two-point autocorrelation functions with those inferred via GFDT from the numerically obtained response function data of $\chi_{r r}$ and $\chi_{\phi \phi}$ (orange crosses). The predicted correlation functions agree with those directly measured from the Hopf oscillator simulations for both the radial and phase fluctuations. Overlaid on all four plots are the respective theoretical calculations (in black lines) from Eqs. (14) and (16).

we show results obtained from the Hopf system with a stateindependent drive, i.e., one with $b^{\prime \prime}=0$. This system, whose drive is independent of its state, admits the GFDT. In Fig. 14, we show that the response (black lines) and fluctuations (colored dots) agree as expected from the GFDT, or the FDT in the Frenet frame that is co-moving with the mean probability current of the driven oscillator.

The fluctuations in the normal (radial) direction (blue) are still well described by a simple Lorentzian (black), whose corner frequency is once again marked by a vertical line. However, the phase diffusion constant exhibits no frequency dependence (red), consistent with Eq. (14). Furthermore, the cross correlations $C_{r \phi}$ vanish, and the correlation data depicted in Figs. 14(c) and 14(d) agree with those inferred from GFDT
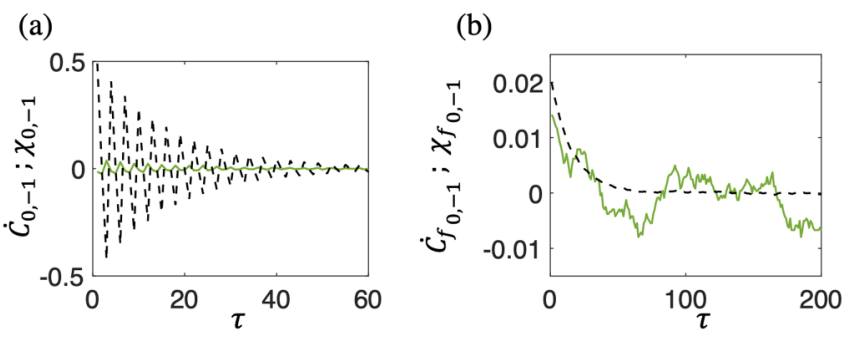

FIG. 15. $\dot{C}_{0,-1}$ (black dashed) vs $\chi(\tau)_{0,-1}$ (green solid) for $p_{\text {curr }}=1$. (a) The time derivative of the numerically computed crosscorrelation function $\dot{C}_{0,-1}$ and the linear response function of $\chi_{0,-1}$ disagree, revealing the breakdown of FDT. (b) However, the system satisfies GFDT, as seen when comparing these functions calculated in the Frenet frame. 
(a)

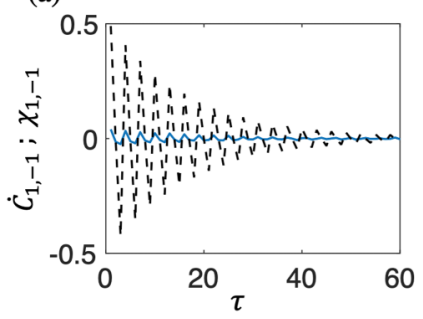

(b)

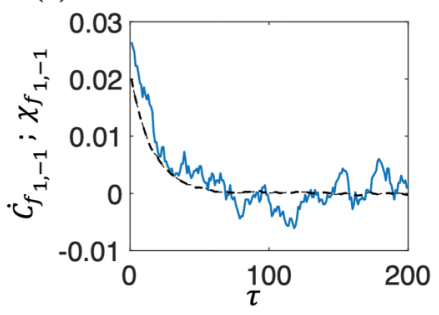

FIG. 16. $\dot{C}_{1,-1}$ (black dashed) vs $\chi(\tau)_{1,-1}$ (blue solid) for $p_{\text {curr }}=$ 1. Comparing the time derivative of the cross-correlation $\dot{C}_{1,-1}$ and response $\chi_{1-1}$ functions, we observe (a) significant deviations from FDT and (b) the satisfaction of GFDT.

and the numerically computed response functions. When $b^{\prime \prime}=$ 0 , the hair cell model violates FDT but obeys GFDT.

For the stochastic discrete-time three-state system with a constant $p_{\text {curr }}$ (Fig. 5), we have shown in the main text for state -1 that the FDT breaks down, but the GFDT is satisfied. In Figs. 15 and 16, we illustrate the same for the other two states, where we obtain similar results.

When examining the second continuous-time three-state system (Fig. 9) with broken detailed balance, but no state dependence, we found that the GFDT holds, as expected. In the main text, we demonstrated the necessary correspondence for only one correlation function-see Fig. 11. For completeness, here we show the analogous results for states -1 and 0 in Figs. 17 and 18, respectively. In all of these examples, the standard FDT breaks down, but the GFDT relations are valid.

\section{APPENDIX E: TRANSITION PROBABILITIES FOR THE FIRST THREE-STATE MODEL}

In Fig. 5, we illustrate our first discrete-time, three-state model whose probability current $p_{\text {curr }}$ can take only the values $\{0,1,2\}$. The corresponding transition probabilities for the purely diffusive system and for a driven system with a constant nonzero probability current are given in Table I.

The work done by the drive generating the constant probability current $p_{\text {curr }}=1$ in this three-state system may be visualized using Fig. 19, where the three states form a helix that is continually descending in energy as the particle traverses the loop of states in an anticlockwise direction. The

(a)
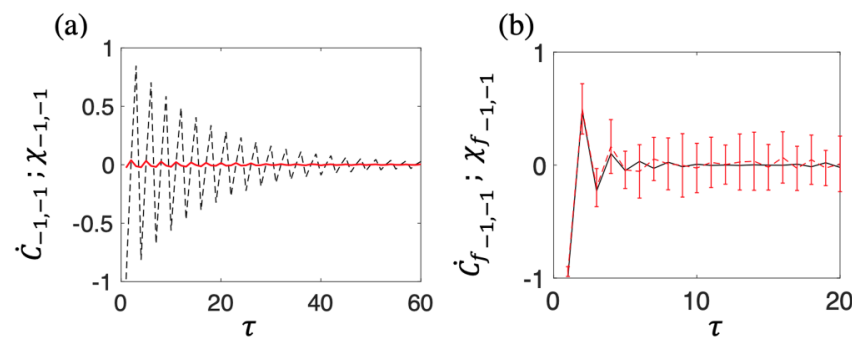

FIG. 17. $\dot{C}$ (black dashed) vs $\chi(\tau)$ (red solid) for $\alpha=98$ and state -1 . (a) Time derivative of the cross-correlation $\dot{C}_{-1-1}$ and the response $\chi_{-1-1}$ functions superimposed, illustrating the breakdown of FDT. (b) The Frenet frame formalism allows for the obedience of GFDT.
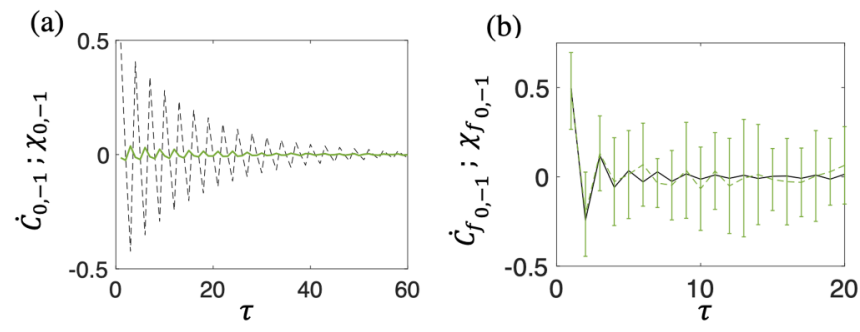

FIG. 18. $\dot{C}$ (black dashed) vs $\chi(\tau)$ (green solid) for $\alpha=98$ for state 0 . Time derivative of the cross-correlation $\dot{C}_{0-1}$ and response $\chi_{0-1}$ functions, demonstrating the (a) violation of FDT and (b) validity of GFDT.

effect of the drive can be thought of as a constantly decreasing gravitational potential as the particle descends the helix, but in order for this to make sense, we must associate the states $k, k^{\prime}, k^{\prime \prime}, k^{\prime \prime \prime}$, where $k=-1,0,1$.

\section{APPENDIX F: LINEAR REGIME OF THE EQUILIBRIUM THREE-STATE MODEL}

In Fig. 10, we perturb the system using an $\epsilon_{-1}$ value of 3 . To verify that the response of this forced oscillator is within its linear regime, in Fig. 20(a) we plot, over a range of $\epsilon_{-1}$ values $(2.6,2.8,3,3.2$, and 3.4), their respective response functions: $\chi_{-1,-1}$. Additionally, in Fig. 20(b), we show that the magnitude of these response functions at time $\tau_{1}$ varies with $\epsilon_{-1}$ in a linear fashion.

\section{APPENDIX G: THREE-STATE SYSTEM WITH A STATE-DEPENDENT DRIVE}

In Fig. 8, we depicted the effects of a state-dependent drive only for the state -1 , namely, the violation of both FDT and GFDT. We obtain similar plots for both states 0 and 1; see Figs. 21 and 22.

\section{APPENDIX H: PROBABILITY CURRENT IN THE SECOND THREE-STATE MODEL}

We rewrite the master equation in Eq. (25) in a form that resembles the Fokker-Planck formalism such that it is comprised of two terms - one is a probability current and the other

TABLE I. Transition probabilities for the three-state model of Fig. 5 .

\begin{tabular}{lccc}
\hline \hline Transitions & $\begin{array}{c}\text { No } \\
\text { drive }\end{array}$ & $\begin{array}{c}\text { Constant drive } \\
\text { with } p_{\text {curr }}=1\end{array}$ & $\begin{array}{c}\text { Constant drive } \\
\text { with } p_{\text {curr }}=2\end{array}$ \\
\hline$-1 \rightarrow-1$ & $1-2 p$ & $p$ & $p$ \\
$-1 \rightarrow 0$ & $p$ & $1-2 p$ & $p$ \\
$-1 \rightarrow 1$ & $p$ & $p$ & $1-2 p$ \\
$0 \rightarrow-1$ & $p$ & $p$ & $1-2 p$ \\
$0 \rightarrow 0$ & $1-2 p$ & $p$ & $p$ \\
$0 \rightarrow 1$ & $p$ & $1-2 p$ & $p$ \\
$1 \rightarrow-1$ & $p$ & $1-2 p$ & $p$ \\
$1 \rightarrow 0$ & $p$ & $p$ & $1-2 p$ \\
$1 \rightarrow 1$ & $1-2 p$ & $p$ & $p$ \\
\hline \hline
\end{tabular}




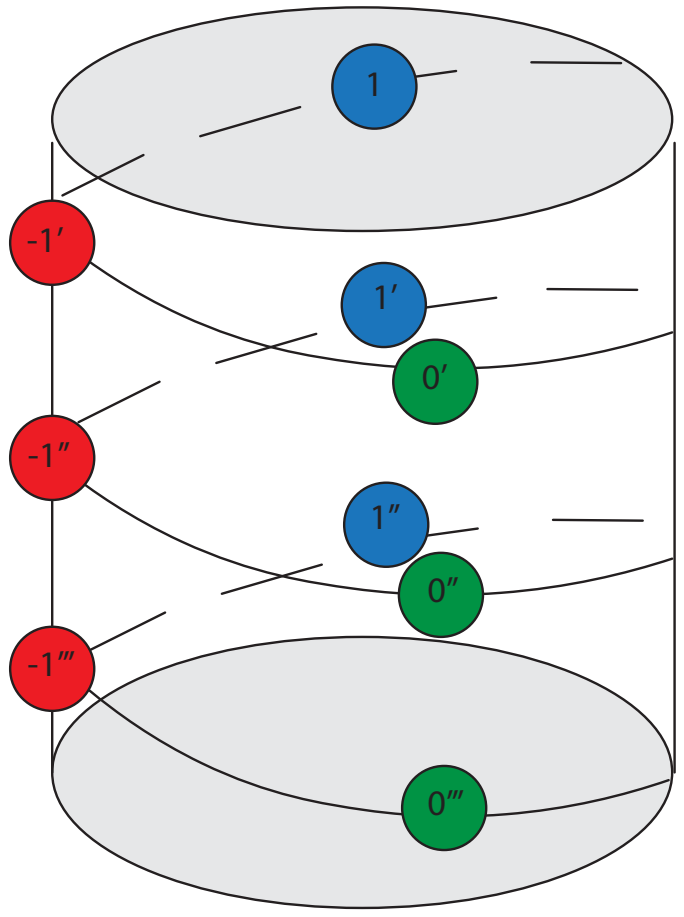

FIG. 19. A schematic diagram illustrating the work done by an internal drive generating constant $p_{\text {curr }}=1$ as moving down a potential. The three-state system is reenvisioned as a repeating set of states on a helix lying on a cylinder in a uniform gravitational potential. The states $\left\{-1^{\prime}, 0^{\prime}, 1^{\prime}\right\}$ are equivalent to the ones below them $\left\{-1^{\prime \prime}, 0^{\prime \prime}, 1^{\prime \prime}\right\}$. The drive is implemented by the change in potential and the mapping rules that relate states $1,1^{\prime}$, and $1^{\prime \prime}$, as shown in the figure.

is diffusive. Let $q$ be the particle's hopping probability (rate of jumping from one state to the neighboring states) and $s$ be the strength of the current. Then, for state 0 , we have

$$
\begin{aligned}
\dot{p}_{0}\left(t_{i}\right)= & p_{-1}\left(t_{i}\right) \alpha_{-10}\left(t_{i}\right)+p_{1}\left(t_{i}\right) \alpha_{10}\left(t_{i}\right) \\
& -p_{0}\left(t_{i}\right) \alpha_{0-1}\left(t_{i}\right)-p_{0}\left(t_{i}\right) \alpha_{01}\left(t_{i}\right) .
\end{aligned}
$$

Using Eq. (26), we can rewrite it as

$$
\begin{aligned}
\dot{p}_{0}\left(t_{i}\right)= & p_{-1}\left(t_{i}\right) \alpha_{-10}+p_{1}\left(t_{i}\right) \alpha_{01} \alpha\left(t_{i}\right) \\
& -p_{0}\left(t_{i}\right) \alpha_{-10} \alpha\left(t_{i}\right)-p_{0}\left(t_{i}\right) \alpha_{01} .
\end{aligned}
$$

(a)
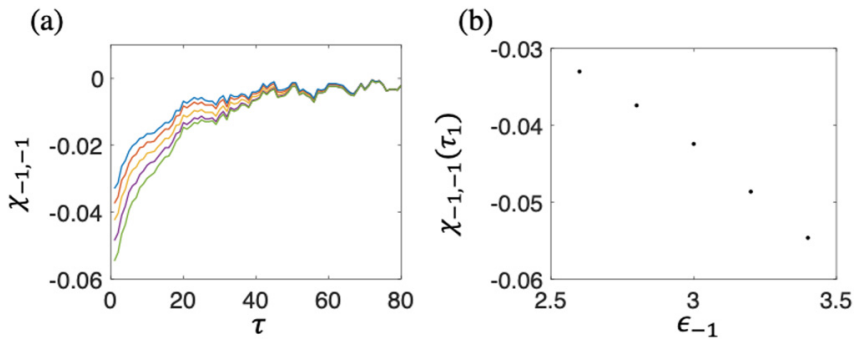

FIG. 20. Variation of $\chi_{-1,-1}$ with $\epsilon_{-1}$. (a) With the three-state model obeying detailed balance, we obtain its $\chi_{-1,-1}$ response by setting the energy of state $-1, \epsilon_{-1}=[2.6,2.8,3,3.2,3.4]$ for one time step. These are, respectively, colored blue, orange, yellow, purple, and green. (b) The magnitude of the $\chi_{-1,-1}\left(\tau_{1}\right)$ values linearly increases with $\epsilon_{-1}$. (a)

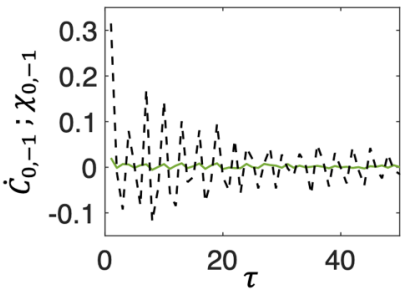

(b)

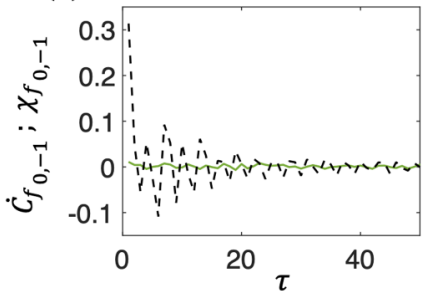

FIG. 21. $\dot{C}_{0,-1}$ (black dashed) vs $\chi(\tau)_{0,-1}$ (green solid) for history-dependent $p_{\text {curr }}$. The time derivative of the cross-correlation function $\dot{C}_{0,-1}$ and the response function $\chi_{0,-1}$, when juxtaposed, reveals the breakdown of (a) FDT and (b) GFDT.

Since the counterclockwise transition rates in Fig. 9 are always 1 , we further simplify this as

$$
\begin{aligned}
\dot{p_{0}}\left(t_{i}\right) & =p_{-1}\left(t_{i}\right)+p_{1}\left(t_{i}\right) \alpha\left(t_{i}\right) \\
& -p_{0}\left(t_{i}\right) \alpha\left(t_{i}\right)-p_{0}\left(t_{i}\right) .
\end{aligned}
$$

Thus, the values of $q$ and $s$ can be computed using the equivalence

$$
\begin{gathered}
p_{-1}\left(t_{i}\right)+p_{1}\left(t_{i}\right) \alpha\left(t_{i}\right)-p_{0}\left(t_{i}\right) \alpha\left(t_{i}\right)-p_{0}\left(t_{i}\right) \\
=q\left(p_{1}-2 p_{0}+p_{-1}\right)+s\left(p_{1}-p_{-1}\right) .
\end{gathered}
$$

So, we obtain by inspection, the following hopping probability $q$ and drive term $s$ :

$$
\begin{aligned}
& q=\frac{\alpha\left(t_{i}\right)+1}{2}, \\
& s=\frac{\alpha\left(t_{i}\right)-1}{2} .
\end{aligned}
$$

Thus, in analogy to the continuous-time Hopf oscillator of Appendix $\mathrm{C}$, the local mean velocity of the deterministic driven three-state system is $\frac{\alpha-1}{2}$. We choose the value of its Frenet frame's velocity accordingly. (a)

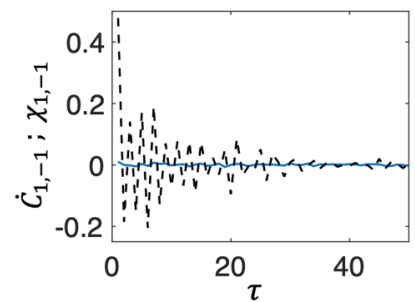

(b)

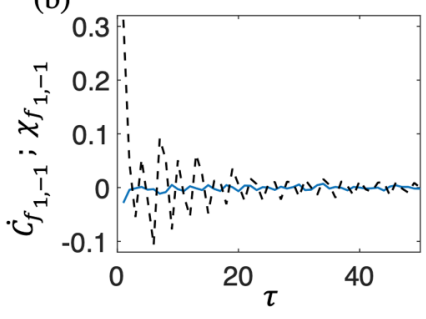

FIG. 22. $\dot{C}_{1,-1}$ (black dashed) vs $\chi(\tau)_{1,-1}$ (blue solid) for history-dependent $p_{\text {curr }}$. Comparison of the time derivative of the cross-correlation $\dot{C}_{1,-1}$ and response $\chi_{1-1}$ functions exemplifies violation of both the (a) equilibrium and (b) generalized fluctuationdissipation relations. 
[1] Biological Clocks: Cold Spring Harbor Symposium on Quantitative Biology 25, edited by E. Bunning (SIAM, New York, 1960).

[2] A. Goldbeter, A model for circadian oscillations in the drosophila period protein (PER), Proc. R. Soc. London B 261, 319 (1995).

[3] T. Mori, B. Binder, and C. H. Johnson, Circadian gating of cell division in cyanobacteria growing with average doubling times of less than 24 hours, Proc. Natl. Acad. Sci. 93, 10183 (1996).

[4] A. Goldbeter, Computational approaches to cellular rhythms, Nature (London) 420, 238 (2002).

[5] E. M. Izhikevich, Dynamical Systems in Neuroscience: The Geometry of Excitability and Bursting, 1st ed. (MIT Press, Boston, 2007).

[6] D. J. Schwab, R. F. Bruinsma, J. L. Feldman, and A. J. Levine, Rhythmogenic neuronal networks, emergent leaders, and Kcores, Phys. Rev. E 82, 051911 (2010).

[7] A. J. Hudspeth, Making an effort to listen: Mechanical amplification in the ear, Neuron 59, 530 (2008).

[8] T. Reichenbach and A. J. Hudspeth, The physics of hearing: Fluid mechanics and the active process of the inner ear, Rep. Prog. Phys. 77, 076601 (2014).

[9] P. Martin, and A. J. Hudspeth, Active hair-bundle movements can amplify a hair cell's response to oscillatory mechanical stimuli, Proc. Natl. Acad. Sci. 96, 14306 (1999).

[10] M. LeMasurier and P. Gillespie, Hair-cell mechanotransduction and cochlear amplification, Neuron 48, 403 (2005).

[11] M. Vollrath, K. Kwan, and D. Corey, The micromachinery of mechanotransduction in hair cells, Ann. Rev. Neurosci. 30, 339 (2007).

[12] R. A. Eatock, Adaptation in hair cells, Annu. Rev. Neurosci. 23, 285 (2000).

[13] S. Camalet, T. Duke, F. Jülicher, and J. Prost, Auditory sensitivity provided by self-tuned critical oscillations of hair cells, Proc. Natl. Acad. Sci. 97, 3183 (2000).

[14] P. G. Gillespie, and J. L. Cyr, Myosin-1c, the hair cell's adaptation motor, Annu. Rev. Physiol. 66, 521 (2004).

[15] P. Martin, A. D. Mehta, and A. J. Hudspeth, Negative hairbundle stiffness betrays a mechanism for mechanical amplification by the hair cell, Proc. Natl. Acad. Sci. 97, 12026 (2000).

[16] M. Benser, R. Marquis, and A. Hudspeth, Rapid, active hair bundle movements in hair cells from the bullfrog's sacculus, J. Neurosci. 16, 5629 (1996).

[17] L. K. Nguyen, Regulation of oscillation dynamics in biochemical systems with dual negative feedback loops, J. R. Soc. Interface 9, 1998 (2012).

[18] G. Lan, P. Sartori, S. Neumann, V. Sourjik, and Y. Tu, The energy-speed-accuracy tradeoff in sensory adaptation, Nat. Phys. 8, 422 (2012).

[19] B. Nadrowski, P. Martin, and F. Jülicher, Active hairbundle motility harnesses noise to operate near an optimum of mechanosensitivity, Proc. Natl. Acad. Sci. 101, 12195 (2004).

[20] S. W. F. Meenderink, P. Quiñones, and D. Bozovic, Voltagemediated control of spontaneous bundle oscillations in saccular hair cells, J. Neurosci. 35, 14457 (2015).
[21] P. Läuger, Shot noise in ion channels, Biochem. Biophys. Acta Biomembr. 413, 1 (1975).

[22] P. Martin, D. Bozovic, Y. Choe, and A. J. Hudspeth, Spontaneous oscillation by hair bundles of the bullfrog's sacculus, J. Neurosci. 23, 4533 (2003).

[23] R. M. Amro and A. B. Neiman, Effect of bidirectional mechanoelectrical coupling on spontaneous oscillations and sensitivity in a model of hair cells, Phys. Rev. E 90, 052704 (2014).

[24] J. Sheth, S. W. F. Meenderink, P. M. Quinones, D. Bozovic, and A. J. Levine, Nonequilibrium limit-cycle oscillators: Fluctuations in hair bundle dynamics, Phys. Rev. E 97, 062411 (2018).

[25] V. M. Eguíluz, M. Ospeck, Y. Choe, A. J. Hudspeth, and M. O. Magnasco, Essential Nonlinearities in Hearing, Phys. Rev. Lett. 84, 5232 (2000).

[26] A. J. Hudsepth, Integrating the active process of hair cells with cochlear function, Nat. Rev. Neurosci. 15, 600 (2014).

[27] H. B. Callen, and T. A. Welton, Irreversibility and generalized noise, Phys. Rev. 83, 34 (1951).

[28] D. Mizuno, C. Tardin, C. F. Schmidt, and F. C. MacKintosh, Nonequilibrium mechanics of active cytoskeletal networks, Science 315, 370 (2007).

[29] T. Speck, and U. Seifert, Restoring a fluctuation-dissipation theorem in a nonequilibrium steady state, Europhys. Lett. 74, 391 (2006).

[30] U. Seifert, Stochastic thermodynamics, fluctuation theorems and molecular machines, Rep. Prog. Phys. 75, 126001 (2012).

[31] U. Seifert, and T. Speck, Fluctuation-dissipation theorem in nonequilibrium steady states, Eur. Phys. Lett. 89, 10007 (2010).

[32] J. R. Gomez-Solano, A. Petrosyan, S. Ciliberto, R. Chetrite, and K. Gawçdzki, Experimental Verification of A Modified Fluctuation-Dissipation Relation for a Micron-Sized Particle in a Nonequilibrium Steady State, Phys. Rev. Lett. 103, 040601 (2009).

[33] C. Kwon, P. Ao, and D. Thouless, Structure of stochastic dynamics near fixed points, Proc. Natl. Acad. Sci. USA 102, 13029 (2005).

[34] M. Baiesi, C. Maes, and B. Wynants, Fluctuations and Response of Nonequilibrium States, Phys. Rev. Lett. 103, 010602 (2009).

[35] M. Baiesi, C. Maes, and B. Wynants, Nonequilibrium linear response for Markov dynamics, I: Jump processes and overdamped diffusions, J. Stat. Phys. 137, 1094 (2009).

[36] G. Verley, K. Mallick, and D. Lacoste, Modified fluctuationdissipation theorem for non-equilibrium steady states and applications to molecular motors, Europhys. Lett. 93, 10002 (2011).

[37] P. Martin, A. J. Hudspeth, and F. Jülicher, Comparison of a hair bundle's spontaneous oscillations with its response to mechanical stimulation reveals the underlying active process, Proc. Natl. Acad. Sci. 98, 14380 (2001).

[38] F. S. Gnesotto, F. Mura, J. Gladrow, and C. P. Broedersz, Broken detailed balance and non-equilibrium dynamics in living systems: A review, Rep. Prog. Phys. 81, 066601 (2018).

[39] J. Sheth, D. Bozovic, and A. J. Levine, Noise-induced distortion of the mean limit cycle of nonlinear oscillators, Phys. Rev. E 99, 062124 (2019). 
[40] L. Dinis, P. Martin, J. Barral, J. Prost, and J. F. Joanny, Fluctuation-Response Theorem for the Active Noisy Oscillator of the Hair-Cell Bundle, Phys. Rev. Lett. 109, 160602 (2012).
[41] J. Prost, J. F. Joanny, and J. M. R. Parrondo, Generalized Fluctuation-Dissipation Theorem for Steady-State Systems, Phys. Rev. Lett. 103, 090601 (2009).

[42] MATHLAB, ver. R2019a, (The MathWorks, Natick, MA, 2019). 